\title{
Exchange interactions in europium monochalcogenide magnetic semiconductors and their dependence on hydrostatic strain
}

\author{
W. Söllinger, ${ }^{1}$ W. Heiss ${ }^{1}$ R. T. Lechner, ${ }^{1}$ K. Rumpf,${ }^{2}$ P. Granitzer,${ }^{2}$ H. Krenn, ${ }^{2}$ and G. Springholz, 冈 \\ ${ }^{1}$ Institute of Semiconductor and Solid State Physics, \\ Johannes Kepler University, Altenbergerstrasse 69, A-4040 Linz, Austria \\ ${ }^{2}$ Institute of Physics, Experimental Physics Div., \\ Karl-Franzens-University, Universitätsplatz 5, A-8010 Graz, Austria
}

(Dated: September 4, 2018)

\begin{abstract}
The classical Heisenberg model is applied in a Monte Carlo study to investigate the distance dependence of the indirect nearest neighbor (NN) exchange and next-nearest neighbor (NNN) superexchange interaction in $\mathrm{EuO}, \mathrm{EuS}, \mathrm{EuSe}$ and EuTe. For this purpose, first, the dependence of the magnetic ordering temperature, i.e., Curie, respectively, Néel temperature for ferromagnetic and antiferromagnetic ordering on the exchange constants was determined. This was then employed for the analysis of experimental data of hydrostatic pressure experiments. It is shown that all experimental findings, i.e., the strong increase of the critical temperatures, as well as the transition from antiferromagnetic to ferromagnetic ordering for EuTe and EuSe with decreasing lattice parameter is well described by the magnetic Grüneisen law, in which the exchange constants depend on the interatomic distances of the $\mathrm{Eu}$ ions in the form of a power law. According to these calculations, the indirect NN exchange is characterized by a Grüneisen exponent of approximately 20 and the NNN superexchange by an exponent of about 10 for all four europium monochalcogenides. The latter agrees with Bloch's empirical 10/3 law for the volume dependence of superexchange interactions in insulating magnetic materials. The Monte Carlo calculations also yield significantly revised exchange constants for unstrained bulk material because spin fluctuations at non-zero temperatures are taken into account. The strong increase of the exchange constants with decreasing lattice parameter provides room for increasing the Curie temperatures in strained epitaxial structures, which is important for device applications.
\end{abstract}

PACS numbers: 75.10.Hk, 75.30.Et, 75.40.Mg, 75.50.Pp

\section{INTRODUCTION}

The europium monochalcogenides $(\mathrm{Eu} X$, with $X=\mathrm{O}$, $\mathrm{S}, \mathrm{Se}$ or $\mathrm{Te}$ ) are wide band gap magnetic semiconductors with cubic rocksalt crystal structure and increasing lattice constant as $X$ changes from $\mathrm{O}$ to $\mathrm{Te} \mathrm{e}_{1,2,3}$. They are considered to be model substances for Heisenberg magnets with spin ordering dominated by indirect nearest neighbor (NN) exchange $J_{1}$ and next-nearest neighbor (NNN) superexchange $J_{2}$ acting between the $S=7 / 2$ localized magnetic moments of the $\mathrm{Eu}^{2+}$ ions with half filled $4 f$ shells $s^{4}$. Depending on the sign and magnitude of the exchange integrals $J_{1}$ and $J_{2}$, the $\mathrm{Eu} X \mathrm{~s}$ exhibit different magnetic phases below the critical ordering temperature ${ }^{\underline{5}}$. $\mathrm{EuO}^{6}$ and $\mathrm{EuS}^{\underline{\underline{7}}}$ are ferromagnets and $\mathrm{EuTe}^{8}$ is an antiferromagnet. EuSe is at the borderline between ferromagnetic and antiferromagnetic ordering. Thus, it shows metamagnetic behavior ${ }^{9.10,11}$, which is influenced by additional contributions from dipolar interactions and crystalline field anisotropies.

The $\mathrm{Eu} X$ s show several outstanding properties, which makes them an interesting class of materials, both academically and for device applications. In external magnetic fields they exhibit a giant spin-splitting of the conduction band and, consequently, extraordinary large magneto-optical effects. EuSe shows the largest effective $g$-factor $\frac{12}{2}$ of up to 18000 and EuTe the largest magnetic field induced energy shifts of the interband transitions 13 observed in semiconducting materials. Potential applications are spin-filter devices based on $\mathrm{EuO}^{14,15,16}$, EuS17,18,19,20,21,22,23,24 or EuSe ${ }^{25}$ tunnel junctions, which provide spin-polarized electrons due to different barrier heights for electrons in different spin states. Also, a huge Faraday rotation is observed in $\mathrm{Eu} X \mathrm{~s} 26,27,28,29$ due to the spin-splitting of the bands, which results in different refractive indices for left and right circular polarized light. Therefore, EuS/ $\mathrm{EuF}_{2}$ and EuSe films have been used for high resolution magneto-optical imaging of the flux distribution in superconductors ${ }^{30}$. Recent work has also demonstrated that $\mathrm{EuO}$ can be epitaxially grown on silicon ${ }^{31.32}$ and $\mathrm{GaN}^{32}$, which opens new possibilities for device realization. Since the Curie temperature of the $\mathrm{Eu} X$ s can be drastically enhanced by doping $32,33,34, \mathrm{EuO}$ might even become a candidate for practical spintronic device applications.

Introducing strain, either omniaxially through hydrostatic pressure $35,36,37,38,39,40,41,42,43,44,45,46,47,48$ or biaxially through epitaxial strain $11,49,50,51,52,53$ leads to drastic changes in the ordering temperatures in the $\mathrm{Eu} X$ compounds and in some cases even to transitions to different kinds of magnetic ordering. For EuO, hydrostatic pressure was found to increase the ferromagnetic ordering temperature $T_{C}$ from 69 to above $200 \mathrm{~K}^{45}$ and for EuS from 16 to almost $180 \mathrm{~K}^{48}$. Metamagnetic EuSe is transformed to a stable ferromagnet already at moderate hydrostatic pressures above $0.5 \mathrm{GPa}^{40}$ and at higher pressures $T_{C}$ increases from 4.7 to $70 \mathrm{~K}$ at $15 \mathrm{GPa} 48$. EuTe 
remains antiferromagnetic up to $9 \mathrm{GPa}$ with nearly constant Néel temperature $T_{N} \approx 10 \mathrm{~K}$, but then becomes ferromagnetic with a $T_{C}$ increasing up to $28 \mathrm{~K}$ when reaching $17 \mathrm{GPa}^{46}$.

The variations in the magnetic properties of the $\mathrm{Eu} X$ compounds are obviously related to the dependence of the exchange integrals $J_{1}$ and $J_{2}$ on the interatomic distances in the crystal lattice. Already by early theoretical work, the basic trend of the $\mathrm{Eu} X$ compounds from antiferromagnetic (EuTe) to ferromagnetic ordering (EuS and $\mathrm{EuO}$ ) was attributed to a strong increase of the ferromagnetic NN exchange $J_{1}$ with decreasing lattice constant from $a_{0}=6.598 \AA$ for EuTe to $5.144 \AA$ for EuO. For the latter, the magnetic ordering is thus dominated by the positive NN exchange $J_{1}$, whereas for antiferromagnetic EuTe the negative NNN exchange $J_{2}$ dominates. Application of hydrostatic pressures $p$ up to $20 \mathrm{GPa}$, produces similar changes in the lattice parameter of up to $8 \%$ compared to the normal bulk values. As a result, large changes in the ordering temperatures are induced

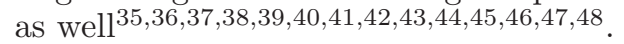

To derive the dependence of the exchange integrals $J_{1}$ and $J_{2}$ on the interatomic distances from hydrostatic pressure experiments, previous works have employed the mean field approximation (MFA) for analysis $46,48,54$. Based on the observation that the Néel temperature $T_{N}$ of antiferromagnetic EuTe does not change appreciably under applied pressure, it was reasoned that the NNN exchange $J_{2}$ is constant in all $\mathrm{Eu} X$ compounds. Therefore, the changes in the magnetic properties were attributed solely to changes in the NN exchange $J_{1}$ and, from the simple mean field expressions, a distance dependence of $J_{1}(a)$ was deduced from the observed changes of $T_{C}(p)$. However, it is well known that the mean field approximation is exact only at zero temperature, i.e., for prediction of the ground state of the system. In particular, the mean field model vastly overestimates the magnetic ordering temperatures due to neglection of spin fluctuations at finite temperatures. As a result, the mean field approximation not only predicts false critical exponents at the phase transition but also much underrated exchange constants.

In the present work, we have employed the Monte Carlo method (MC) to calculate the magnetic phase diagrams and ordering temperatures of the $\mathrm{E} u X$ compounds as a function of the exchange interactions. The MC method takes the mutual interactions between all spins into account and allows for spin fluctuations at $T$ above zero. Using finite size scaling techniques, MC correctly predicts the transition temperatures and the behavior of the order parameters at criticality for a given model Hamiltonian ${ }^{55}$. Moreover, due to the spin fluctuations near the transition temperature in the $\mathrm{MC}$ calculations, the Néel point of antiferromagnetic ordering as in EuTe is found to depend on on the exchange constants $J_{1}$ and $J_{2} \frac{56}{}$, in contrast to the mean field approximation, where the Néel point depends on $J_{2}$ only. Thus,the basic assumption of the previous analyses does not hold $\underline{46,48,54}$.
The experimental data for $\mathrm{Eu} X \mathrm{~s}$ under hydrostatic pressure is therefore reexamined by Monte Carlo calculations and by considering magnetic Grüneisen power laws $\frac{57}{5}$ for the distance dependence of the NN and NNN exchange constants. We show that for the whole family of $\mathrm{Eu} X$ compounds, the distance dependence of the exchange interactions $J_{1}\left(r_{1}\right)$ and $J_{2}\left(r_{2}\right)$ can be consistently described by unique Grüneisen exponents of $n_{1} \approx 20$ and $n_{2} \approx 10$ by which the whole set of experimental data of the $\mathrm{EuX}$ compounds under hydrostatic pressure can well be explained. The obtained exponent of $n_{2} \approx 10$ for $J_{2}$ is also consistent with Bloch's empirical 10/3 law 57 for the volume dependence of the superexchange interaction $J \sim V^{-10 / 3}$ observed for a wide variety of insulating magnetic material systems.

The paper is organized as follows: In Sec. II we present the model Hamiltonian and briefly discuss the technical details of the Monte Carlo calculations. In Sec. III, the method is applied to bulk EuTe under ambient pressure, demonstrating that the whole magnetic phase diagram can be well described and that the exchange constants $J_{1}$ and $J_{2}$ obtained from Monte Carlo calculations strongly differ from literature values derived by the mean field approximation. In Sec. IV the method for determination of the distance dependence of the exchange constants is described and applied to EuTe. Due to the pressure induced transition between antiferromagnetic and ferromagnetic ordering, accurate dependencies for both $J_{1}\left(r_{1}\right)$ and $J_{2}\left(r_{2}\right)$ are determined. The approach is then extended to EuO, EuS and EuSe in Secs. V] and VI, revealing that the same functional behavior, i.e., the same Grüneisen exponents provide an excellent description of the experimental data for all $\mathrm{Eu} X$ compounds. In Sec. VII the results are compared in detail and the applicability of other types of functional dependence of $J_{1}\left(r_{1}\right)$ and $J_{2}\left(r_{2}\right)$ discussed.

\section{DETAILS OF THE CALCULATION}

For the calculation of the magnetic properties of the $\mathrm{Eu} X$ s we employed the classical Heisenberg model with nearest and next nearest neighbor exchange interaction taken into account. The corresponding model Hamiltonian reads as

$$
\mathcal{H}=-\sum_{i \neq j} J_{i j} \boldsymbol{S}_{i} \boldsymbol{S}_{j}-g \mu_{B} \boldsymbol{H} \sum_{i} \boldsymbol{S}_{i}
$$

where $\boldsymbol{H}$ denotes the external magnetic field and $J_{i j}+$ $J_{j i}$ is the total exchange interaction between two spins located at lattice sites $i$ and $j$ and

$$
J_{i j}=\left\{\begin{array}{rll}
J_{1} & : & i \text { is NN of } j \\
J_{2} & : & i \text { is NNN of } j \\
0 & : & \text { else }
\end{array} .\right.
$$

In the Monte Carlo calculations we considered rhombohedral $f c c$ clusters of classical spins, where all clus- 
ter boundaries are (111) lattice planes. This is a convenient choice of geometry, since antiferromagnetic and ferrimagnetic ordering in EuSe and EuTe is comprised of ferromagnetic (111) planes and epitaxial EuTe and EuSe samples are usually grown in (111) orientation $11,12,13,58$. The choice of geometry has, however, no influence on the results of our calculations. Clusters of up to $32^{3}$ spins with periodic boundary conditions were considered. During a single Monte Carlo step, random orientations are generated for every single spin, which are then accepted or rejected according to the Metropolis criterion $\underline{59}$. Observeables like the total energy, the overall magnetization, the magnetization in the direction of the external field, the transverse magnetization and the corresponding staggered magnetic moments are computed after every Monte Carlo step. Simulations were performed with up to $N=10^{5}$ iterations and additional Monte Carlo steps for equilibration at the beginning of every run. For a single simulation the temperature $T$, the external magnetic field $H$ and the number of spins are constant.

The expectation value for the total energy is given by

$$
\langle\mathcal{H}\rangle \approx \frac{\sum_{i=1}^{N} \mathcal{H}_{i} \exp \left(-\beta \mathcal{H}_{i}\right)}{\sum_{i=1}^{N} \exp \left(-\beta \mathcal{H}_{i}\right)},
$$

where $\beta=1 / k_{B} T$. The Metropolis algorithm causes the total energy to be distributed according to Boltzmann's law. Hence, the expectation values for the total energy and for the magnetic moment become arithmetic mean values in the simulation

$$
\begin{gathered}
\langle\mathcal{H}\rangle=\frac{1}{N} \sum_{i=1}^{N} \mathcal{H}_{i} \\
\langle\boldsymbol{M}\rangle=\frac{1}{N} \sum_{i=1}^{N} \boldsymbol{M}_{i}
\end{gathered}
$$

To determine the critical ordering temperatures the fourth-order cumulant of the corresponding order parameter is used, which for ferromagnetic ordering at zero external field is defined as 55

$$
U=1-\frac{\left\langle M^{4}\right\rangle}{3\left\langle M^{2}\right\rangle^{2}}
$$

where $\left\langle M^{2}\right\rangle$ and $\left\langle M^{4}\right\rangle$ denote the second and fourth order moments of the probability distribution of the magnetization. The fourth order cumulants show universal values at the critical temperature. Thus, during simulation $U$ is generated as a function of temperature and recorded for various cluster sizes. The different $U(T)$ curves cross in a single point at the critical temperature, as shown in detail in Sec. III The transition temperatures obtained from our calculations were compared to theoretical predictions from high-temperature series expansions 60 . For ferromagnetic nearest neighbor exchange $\left(J_{1}>0\right)$ and no next-nearest neighbor exchange $\left(J_{2}=0\right)$ between classical spins in the $f c c$ lattice, we obtained a critical temperature $T_{C}$ defined by $2 J_{1} S^{2} /\left(k_{B} T_{C}\right)=0.3149 \pm 0.0008$ in $2 \times 10^{5}$ Monte Carlo steps. The prefactor of 2 stems from the fact, that according to the definition of Eqn. (1) the exchange interaction between pairs of spins is always added twice. Despite the simplicity of our approach compared to other sophisticated Monte Carlo routines61.62, our result is in excellent agreement the theoretically predicted and generally accepted value of Ritchie and Fisher $\underline{\underline{60}}$ of $0.3147 \pm 0.0001$ for this relation.

If the NNN exchange interaction is antiferromagnetic and $\left|J_{2}\right|>J_{1}$, the simulation generates a classical Néel state with eight ferromagnetically ordered sublattices as the ground state $\mathrm{e} \underline{ }$. This is a consequence of the antiferromagnetic ordering degenerating into the four equivalent (111) directions ${ }^{52}$ in the simulation. In this case, four pairs of antiferromagnetically aligned sublattices can rotate freely and the fourth order cumulants are defined as 63

$$
U^{s t}=\frac{5}{2}-\frac{3}{2} \frac{\left\langle\left(M^{s t}\right)^{4}\right\rangle}{\left\langle\left(M^{s t}\right)^{2}\right\rangle^{2}}
$$

for zero field and

$$
U_{\perp}^{s t}=2-\frac{\left\langle\left(M_{\perp}^{s t}\right)^{4}\right\rangle}{\left\langle\left(M_{\perp}^{s t}\right)^{2}\right\rangle^{2}}
$$

for nonzero external field. Here, $M^{s t}$ and $M_{\perp}^{s t}$ denote the staggered (transverse) magnetization, with transverse referring to the component of the magnetization perpendicular to the external field.

$$
\begin{aligned}
& M^{s t}=\sum_{i=1}^{8}\left|\boldsymbol{M}^{(i)}\right| \\
& M_{\perp}^{s t}=\sum_{i=1}^{8}\left|\boldsymbol{M}_{\perp}^{(i)}\right|
\end{aligned}
$$

Eqns. (9) and (10) are sums of the absolute values of the (transverse) magnetization over the eight possible sublattices. Due to additional anisotropies and/or dipolar couplings, this degenerate AFM state is not observed experimentally, but only domains with completely ferromagnetically ordered $\mathrm{Eu}$ (111) planes, which is one possible case in our MC simulations. Here it should be also noted that in a quantum mechanical treatment it has been shown ${ }^{64}$ that a classical Néel state is not an eigenstate of the system. However, Anderson $\frac{65}{5}$ showed that the upper limit of the error introduced by utilizing the classical Heisenberg model is $1 /(Z S)$, where $Z$ is the number of nearest neighbors. Since the Eu-ions carry a relatively large spin of $S=7 / 2$, the error in the ground state energy is smaller than $2.4 \%$. Thus, a classical treatment considering continuously rotating spin vectors is well justified. 


\section{EXCHANGE INTERACTIONS IN EuTe AT AMBIENT PRESSURE}

In our Monte Carlo study of the exchange interactions, EuTe is chosen as test material. This is because detailed experimental data is available for the $H$ - $T$ phase diagram ${ }^{8}$, which allows a direct determination of the NN and NNN exchange interaction $J_{1}$ and $J_{2}$ based on the antiferromagnetic ordering temperature $T_{N}$ and the critical field at zero temperature $H_{C}(T=0)$. In addition, the ferromagnetic $\left(J_{1}\right)$ and the antiferromagnetic exchange $\left(J_{2}\right)$ are relatively balanced in EuTe. Therefore, pronounced changes in magnetic ordering occur when hydrostatic pressure is applied.

\section{A. Experimental Results}

The magnetic properties of EuTe were determined using DC and AC SQUID magnetometry measurements of high quality $4 \mu \mathrm{m}$ thick (111)-oriented epitaxial layers grown by molecular beam epitaxy on $\mathrm{BaF}_{2}$ substrates $13,66,67$. As a result, the magnetization and AC susceptibility was obtained as a function of both temperature and external magnetic field up to $7 \mathrm{~T}$. The external field direction was applied in the (111) growth plane, which is also the easy plane of the magnetization.

Experimental magnetization curves $M(T)$ at different applied external fields from 5.5 to $7 \mathrm{~T}$ are shown in Fig. 1(a). The magnetization curves exhibit clear peaks at the phase transition between the antiferromagnetic and the paramagnetic phase. High magnetic fields were applied in order to obtain information about the critical field at zero temperature $H_{C}(T=0)$. Field-dependent AC susceptibility curves were also measured. As shown in Fig. 1(b), for $T=5 \mathrm{~K}$ the susceptibility is essentially constant below the critical field, corresponding to a linear increase in the magnetization. This arises from the continuous reduction of the relative angle between the spins in the adjacent (111) lattice planes from $180^{\circ}$ in the AFM II state at zero field to almost zero at the critical field $H_{C}$, as illustrated schematically by the arrows in Fig. 1(e). Thereby the spin orientation changes from initially perpendicular to the external field to finally parallel to the external field direction when $H$ reaches the critical field. The critical field is thus given by the discontinuous drop in the susceptibility at this point (see Fig. 1(b)).

Figure 1(c) displays the susceptibility as a function of temperature at zero external field, showing a broad peak with a maximum slightly above $10 \mathrm{~K}$. However, the phase transition from antiferromagnetic to paramagnetic corresponds to the peak in the specific heat, which according to Fisher's rule ${ }^{68}$ coincides with the maximum slope, i.e., the inclination point in the AC susceptibility. As indicated by the arrow in Fig. 1(c), the phase transition thus occurs at $T_{N}=9.85 \pm 0.05 \mathrm{~K}$.

Figure 1(d) shows the phase diagram of EuTe compiled
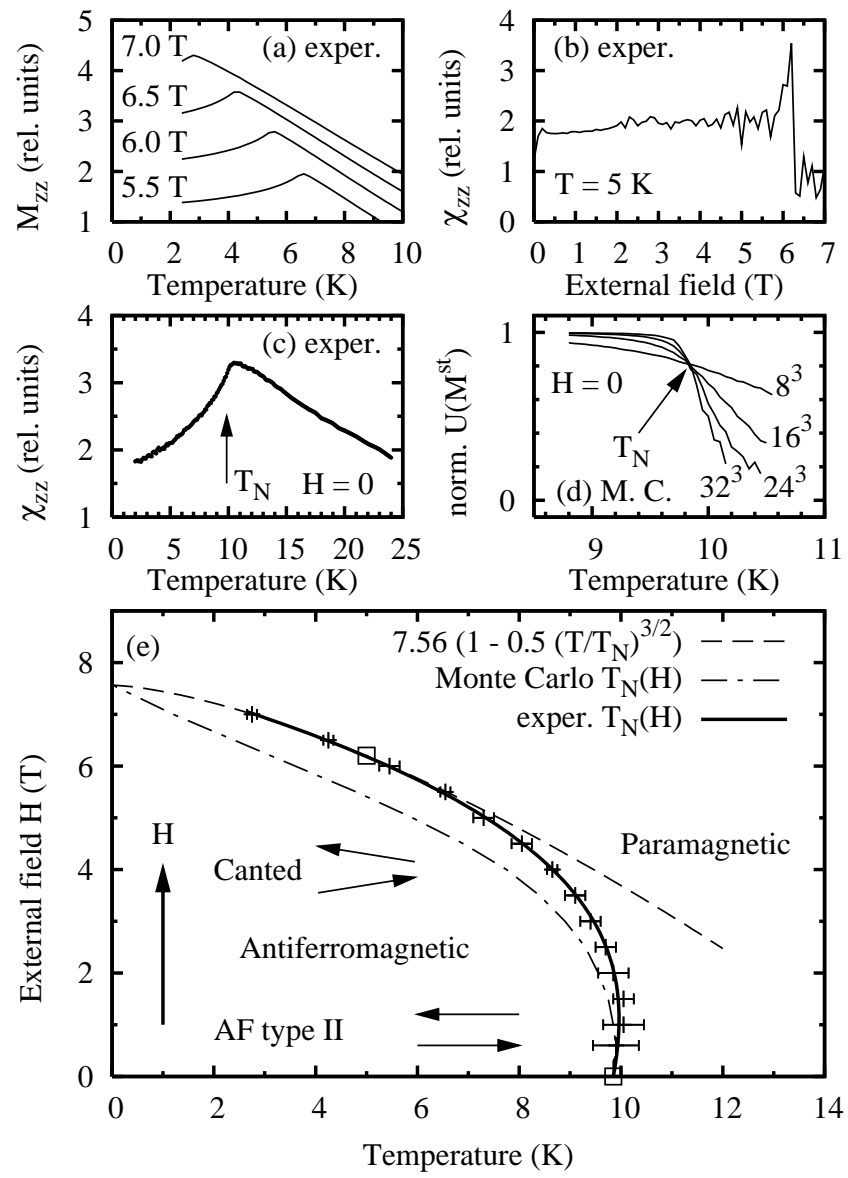

FIG. 1: Magnetic properties of bulk EuTe at normal pressure derived from SQUID measurements and Monte Carlo simulations. (a) Temperature-dependent magnetization $M(T)$ measured for various in plane external magnetic field values from 5.5 to 7 T. (b) AC susceptibility vs. external magnetic field at $5 \mathrm{~K}$ indicating the critical field as discontinuity at $B \approx 6.2 \mathrm{~T}$. (c) AC susceptibility $\chi$ measured as a function of temperature at zero external field. The inclination point indicated by the arrow yields a Néel temperature of $9.85 \pm 0.05$ K. (d) Determination of the Néel temperature from Monte Carlo data using the fourth order cumulant of the staggered magnetization $U\left(M^{s t}\right)$ for different system sizes. The curves show a common intersection at the Néel temperature. (e) Phase diagram of EuTe: Symbols with error bars correspond to maxima or inflection points in $M(T)$ for high, respectively, small external fields; squares to the discontinuity in $\chi(H)$ or the inflection point in $\chi(T)$. The solid line indicates the experimental $H_{C}(T)$ phase boundary and the dash-dotted line represents the boundary obtained by the Monte Carlo calculations. The $T^{3 / 2}$ extrapolation (dashed line) of the experimental data towards $T=0$ yields a critical field of $H_{C}=7.56 \mathrm{~T}$.

from the experimental data (symbols and solid line), where the thick solid line indicates the experimentally determined $H_{C}(T)$ phase boundary. A $T^{3 / 2}$ curve, which according to spin wave theory is the low-temperature behavior of the critical field, was fitted to the experimental 


\begin{tabular}{lccc}
\hline \hline Reference & Exper. results / Analysis & $J_{1}(\mathrm{~K})$ & $J_{2}(\mathrm{~K})$ \\
\hline Oliveira $^{8}$ & $H_{C}, \theta / \mathrm{MFA}$ & 0.100 & -0.215 \\
Zinn $^{1}$ & $H_{C}, \theta / \mathrm{MFA}$ & 0.060 & -0.200 \\
Wachter $^{2}$ & $H_{C}, T_{N} / \mathrm{MFA}$ & 0.043 & -0.150 \\
Köbler $^{69}$ & $H_{C}, T_{N} / \mathrm{MFA}$ & 0.060 & -0.160 \\
Kuneš $^{70}$ & ab initio LDA $+U(U=6 \mathrm{eV})$ & 0.110 & -0.320 \\
\hline Our study & $H_{C}, T_{N} / \mathrm{MFA}$ & 0.035 & -0.156 \\
& $H_{C}, T_{N} / \mathrm{MC}$ & 0.192 & -0.313 \\
\hline \hline
\end{tabular}

TABLE I: Comparison of the exchange constants $J_{1} / k_{B}$ and $J_{2} / k_{B}$ of EuTe determined by the analysis of experimental data for the Néel temperature $T_{N}$, the critical field $H_{C}$ or the paramagnetic Curie temperature $\theta_{C}$ using the mean field approximation (MFA) or the Monte Carlo (MC) method (present work). Also listed are the exchange constants derived by Kuneš et al. from ab initio calculations.

critical points between $2 \mathrm{~K}$ and $6 \mathrm{~K}$, yielding

$$
H_{C}(T)=H_{C}(0)\left[1-\lambda\left(T / T_{N}\right)^{3 / 2}\right] .
$$

Extrapolation of the the measured $H_{C}(T)$ to $T=0$ thus yields the critical field at zero temperature of $H_{C}(0)=$ $7.56 \pm 0.02 \mathrm{~K}$. The dashed line in Fig. 1(e) represents Eqn. (11) with the coefficient $\lambda=0.50 \pm 0.01$. The experimental data as well as $T_{N}, H_{C}(0)$ and $\lambda$ are in excellent agreement with previous results of Oliveira et al.

\section{B. Exchange constants and phase diagram from MC calculations}

In most previous studies $1,2,8,69$, the mean field analysis was used to determine the exchange integrals in EuTe, because it provides simple analytic expressions for the critical field at zero temperature $H_{C}(0)$, the Néel temperature $T_{N}$ as well as the paramagnetic Curie temperature $\theta_{C}$ as a function of $J_{1}$ and $J_{2}$. For type IIantiferromagnetic ordering in an $f c c$ lattice with $\mathrm{NN}$ and NNN exchange interactions, the mean field approximation (MFA) yields

$$
\begin{aligned}
H_{C}^{\mathrm{MFA}}(0) & =-4 S\left(6 J_{1}+6 J_{2}\right) /\left(g \mu_{B}\right), \\
T_{N}^{\mathrm{MFA}} & =\frac{2}{3} S(S+1)\left(-6 J_{2}\right) / k_{B}, \text { and } \\
\theta_{C}^{\mathrm{MFA}} & =\frac{2}{3} S(S+1)\left(12 J_{1}+6 J_{2}\right) / k_{B},
\end{aligned}
$$

where $g=2$ and $S=7 / 2$ for the magnetic moment of the $\mathrm{Eu}^{2+}$ ions. Inserting our experimental values for $H_{C}(0)$ and $T_{N}$ into Eqns. (12) and (13) and solving for $J_{1}$ and $J_{2}$ yields $J_{1}^{\mathrm{MFA}} / k_{B}=0.035 \mathrm{~K}$ and $J_{2}^{\mathrm{MFA}} / k_{B}=-0.156 \mathrm{~K}$. As shown in Tab. I, the values are consistent with previous mean field studies, especially those, which applied the same analysis of the experimental $H_{C}(0)$ and $T_{N}$ based on MFA Eqns. (12) and (13).

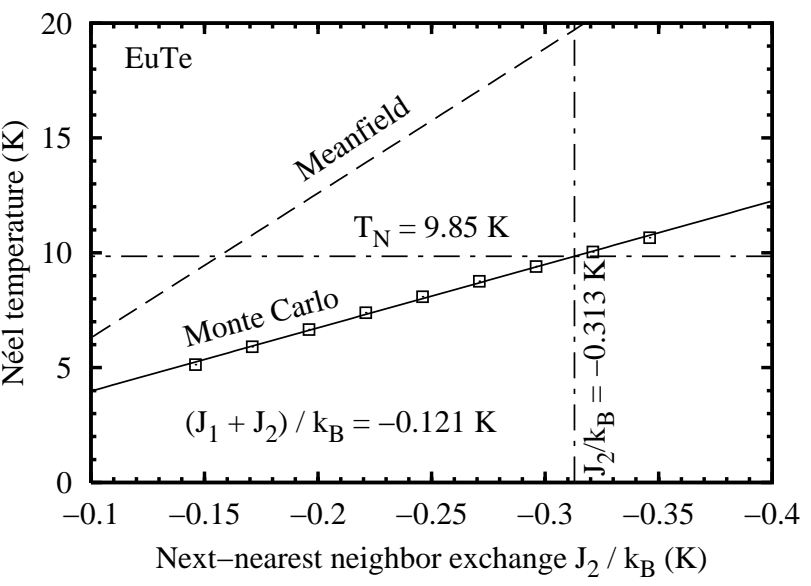

FIG. 2: Néel temperature of EuTe as a function of the nearest neighbor exchange constant $J_{1}$ under the constraint $\left(J_{1}+J_{2}\right) / k_{B}=-0.121 \mathrm{~K}$, which is a consequence of evaluating the experimental critical field at zero temperature $H_{C}(T=0)$ - see in the text for details. We obtain a linear function (squares and solid line) which is shown in comparison with the corresponding mean field relation (dashed line). At $J_{2}=-0.313 \mathrm{~K}\left(J_{1}=0.192 \mathrm{~K}\right)$ the Monte Carlo curve reaches the experimental Néel point of $9.85 \mathrm{~K}$.

In the Monte Carlo calculations, the transition temperature $T_{N}(0)\left(T_{N}(H \neq 0)\right)$ is deduced from the temperature dependence of the $4^{\text {th }}$ order cumulants of the staggered (transverse) magnetization $U_{(\perp)}^{s t}$. As described in Sec. II these cumulants show universal values at the critical temperature $T_{N}$ independent of cluster size. This is demonstrated in Fig. 11 (d) for the case of $H=0$. Using the exchange constants $J_{1}^{M F A}$ and $J_{2}^{M F A}$ derived from the mean field analysis, the Monte Carlo calculations yield a Néel temperature of $5.45 \mathrm{~K}$ at zero external field, which is in strong disagreement with the experimental value of $9.85 \mathrm{~K}$. This clearly demonstrates that the neglection of spin fluctuations in MFA leads to a vast underestimation of the exchange constants, an effect that has been already noted in previous theoretical studies 56,63 . In the MC calculations, moreover, the critical Néel temperature $T_{N}$ is found to depended significantly not only on the antiferromagnetic exchange constant $J_{2}$ but also on the ferromagnetic NN exchange constant $J_{1}$, in contrast to the MFA approximation where $T_{N}$ depends only on the antiferromagnetic exchange - see Eqn. (13). This is due to the fact that in the mean field approximation, for type II antiferromagnetic ordering the $J_{1}$ exchange between the $6 \mathrm{NN} \mathrm{Eu}^{2+}$ ions within the ferromagnetic (111) planes exactly cancels with the $J_{1}$ exchange to the $6 \mathrm{NN} \mathrm{Eu}^{2+}$ ions within the antiferromagnetically coupled neighboring (111) lattice planes. This does not apply for the MC calculations because of the non perfect antiferromagnetic spin alignment at nonzero temperatures that results from spin fluctuations.

In order to determine the exchange constants from the Monte Carlo calculations, we have systematically calcu- 
lated the Néel temperature as a function of both exchange constants $J_{1}$ and $J_{2}$. As a boundary condition, we take advantage of the fact that in the limit of $T=0$, the critical field $H_{C}$ of the Monte Carlo calculations converges exactly to the mean field value of Eqn. (12). This is because spin fluctuations are absent at $T=0$, and thus the MFA represents the exact ground state of the system. Therefore, the experimental value of $H_{C}(T=0)$ and Eqn. (12) determine the sum of the exchange constants as

$$
\left(J_{1}+J_{2}\right) / k_{B}=-0.121 \mathrm{~K} .
$$

in EuTe. This eliminates one independent variable, i.e., with this condition, only $J_{2}$ must be varied for the calculation of $T_{N}$. The resulting dependence is plotted in Fig. 2 (squares and solid line). Evidently, $T_{N}$ varies almost perfectly linearly and can be represented by the relation

$$
\left.T_{N}^{\mathrm{MC}}\right|_{\left(J_{1}+J_{2}\right) / k_{B}=-0.121 \mathrm{~K}}=1.22-27.57 J_{2} / k_{B}
$$

within the range $-0.35 \leq J_{2} / k_{B} \leq-0.15$. Solving Eqn. (16) for $J_{2}$ and inserting the experimental Néel point of bulk EuTe $T_{N}=9.85 \mathrm{~K}$ (horizontal dash-dotted line in Fig. 2) yields $J_{2}^{\mathrm{MC}} / k_{B}=-0.313 \mathrm{~K}$ and hence $J_{1}^{\mathrm{MC}} / k_{B}=0.192 \mathrm{~K}$ from Eqn. (15) as the intrinsic exchange constants of bulk EuTe. It is noted, that calculating $T_{N}$ without the constraint of Eqn. (15) yields a function $T_{N}\left(J_{1}, J_{2}\right)$ that depends nonlinearly on $J_{1}$ and $J_{2}$, in contrast to the MFA Eqn. (13), which predicts only a linear dependence on $J_{2}$ - see Sec. IV for further details. From the MC calculations, the Néel temperature as a function of $J_{1}$ and $J_{2}$ is found to be well described by

$$
\begin{aligned}
T_{N}^{\mathrm{MC}} & \approx\left(-15.3 J_{1}-40.8 J_{2}\right) / k_{B} \\
& =\frac{2}{3} S(S+1)\left(-1.46 J_{1}-3.89 J_{2}\right) / k_{B}
\end{aligned}
$$

in a linear approximation in the vicinity of the intrinsic EuTe exchange constants, demonstrating that the Néel temperature indeed depends strongly on both exchange constants.

As demonstrated by Tab. \, which compares our derived set of exchange constants with previously published ones, our values are nearly twice as large as those derived from mean field analysis. Thus, by neglection of spin fluctuations the exchange parameters are vastly underestimated. Remarkably, the exchange constants derived from our Monte Carlo calculations are in good agreement with recent ab initio calculations of Kuneš et al $\stackrel{70}{.}$ using the local-density-approximation method including strong Coulomb repulsion within the $4 f$ shells $(\mathrm{LDA}+U)$. In particular, our NNN exchange constant $J_{2}$, which in Ref. 70 depends very weakly on the Coulomb parameter $U$ matches the ab initio result very well.

With the new exchange parameters, we can now calculate the whole magnetic phase diagram of EuTe using the

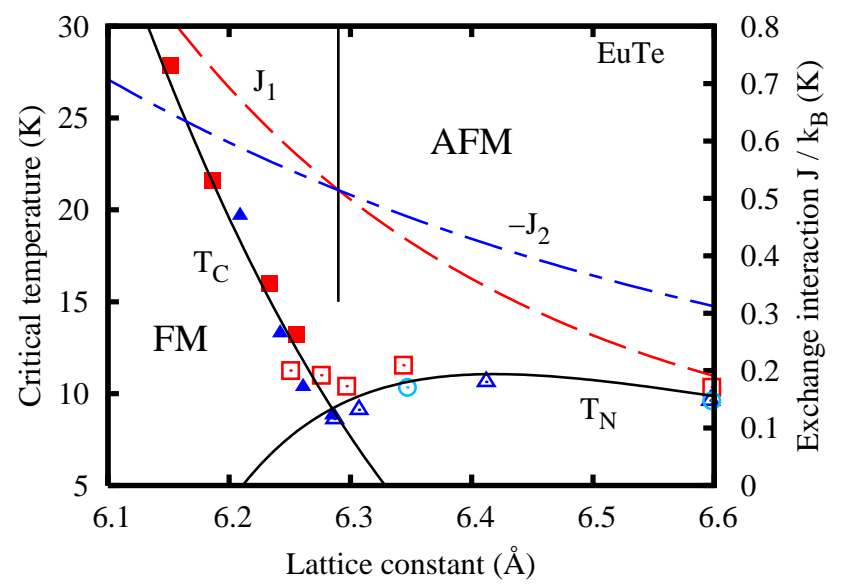

FIG. 3: (color online) Critical temperatures (left scale) and exchange interactions $J_{1}$ and $J_{2}$ (right scale) in EuTe plotted as a function of the lattice constant $a$. Open and closed symbols ( $\operatorname{circles}^{38}$, squares $\frac{46}{}$, triangles $\frac{47}{}$ ), correspond to Néel $\left(T_{N}\right)$, respectively Curie points $\left(T_{C}\right)$ determined from hydrostatic pressure experiments. Solid lines: $T_{N}(a)$ and $T_{C}(a)$ derived from Monte Carlo calculations using a $J_{1}(a)$ and $J_{2}(a)$ dependence given by the Grüneisen law (18) with best fitting power law exponents of $n_{1}=20.6 \pm 0.4$ and $n_{2}=10.4 \pm 0.5$, respectively. The corresponding variation of $J_{1}(a)$ and $J_{2}(a)$ as functions of the lattice parameter is shown as the dashed and dash-dotted line, respectively.

$4^{\text {th }}$ order cumulants of the staggered (transverse) magnetization for various external magnetic fields and cluster sizes. The resulting phase boundary $T_{N}(H)$ is depicted as dash-dotted line in Fig. 1(e). As expected, the calculated $H_{C}(T)$ approaches the experimental value of $7.56 \mathrm{~T}$ in the limit of $T \rightarrow 0$ and the calculations nearly follow the experimental $H_{C}(T)$ boundary. The fact, that at $T>0$ the calculated $H_{C}(T)$ values are slightly lower than the measured ones and that at low temperatures, the calculated critical field varies linearly with temperature instead of obeying a $T^{3 / 2}$ behavior is a well known consequence of applying a classical $(S=\infty)$ instead of the quantum mechanical $S=7 / 2$ model in our calculations. As already noted in Sec. III, the error in the ground state energy introduced by this simplification is of the order of less than $2.4 \%$ for our type of system.

\section{DISTANCE DEPENDENCE OF EXCHANGE INTERACTIONS IN EuTe}

Under hydrostatic pressure, the EuTe lattice constant can be compressed from its normal bulk value of $a_{0}=$ $6.589 \AA$ to about $6.15 \AA$ at a pressure reaching $17 \mathrm{GPa} 46$. This corresponds to a $7 \%$ reduction of the lattice constant and of the interatomic distances of the $\mathrm{Eu}^{2+}$ ions in the crystal, where in the $f c c$ lattice of EuTe the NN Eu ${ }^{2+}$ distance is $r_{1}=a / \sqrt{2}$ and the NNN distance $r_{2}=a$. The resulting changes in the magnetic ordering temperatures 
obtained by hydrostatic pressure experiments $38,46,47$ are compiled in Fig. 3. where the open symbols represent the measured $T_{N}$ and the full symbols the $T_{C}$ values plotted as a function of lattice constant. Since at ambient pressure, the antiferromagnetic exchange $J_{2}$ of EuTe is larger than the ferromagnetic exchange $J_{1}$, a type II antiferromagnetic ordering 5 occurs below the Néel point of $T_{N}$ $=9.85 \mathrm{~K}$. With increasing pressure, i.e., decreasing lattice constant, the Néel temperature remains practically constant at $T_{N} \approx 10 \mathrm{~K}$, but at $\approx 9 \mathrm{GPa}$ or $5 \%$ compressive strain EuTe becomes ferromagnetic $\frac{46,47}{}$ with rapidly increasing Curie $T_{C}$ that rises up to $28 \mathrm{~K}$ at $17 \mathrm{GPa}^{46}$. The observed phase transition from antiferromagnetism to ferromagnetism at $a=6.29 \AA$ implies that at smaller atom distances the NN $J_{1}$ becomes the dominating exchange mechanism.

The influence of the inter-atomic distances $r_{i}$ on exchange constants has been a subject of many theoretical studies $1,4,54,71,72,73$. However, indirect and superexchange mechanisms involve complex integrals such that up to now no general analytic expressions for their distance dependence have been derived theoretically. An empirical power law dependence, referred to as the magnetic Grüneisen law, has been proposed by Bloch ${ }^{57}$, i.e.,

$$
J(r)=J_{0}\left(\frac{r}{r_{0}}\right)^{-n},
$$

where $J_{0}=J\left(r_{0}\right)$ and $r_{0}$ are the exchange interaction and interatomic distance at normal pressure, and $n$ is the scaling exponent. As shown in Ref. 57, this dependence well describes the observations for many magnetic semiconductors or insulators such as the $\mathrm{Mn}$ and $\mathrm{Gd}$ chalcogenides or iron oxides, for which the power law exponent $n$ shows a universal value of around 10 for the magnetic superexchange ${ }^{57}$. This also yields the empirical $10 / 3$ law for the volume dependence of superexchange $e^{57}$ of $J(V)=J_{0}\left(V / V_{0}\right)^{-10 / 3}$.

To test if the Grüneisen dependence of Eqn. (18) adequately describes the atomic distance dependence of the exchange integrals in EuTe, we have performed a series of Monte Carlo calculations of the Néel and Curie temperature as a function of the exchange integrals in order to fit the experimental $T_{N}(a)$ and $T_{C}(a)$ data of Fig. 3 using the power law exponents $n_{1}$ and $n_{2}$ for the NN and NNN exchange interactions $J_{1}$ and $J_{2}$ as free parameters. In these calculations, the ferromagnetic NN exchange $J_{1}>0$ and the antiferromagnetic NNN exchange $J_{2}<0$ were varied independently in the range of $0.190 \leq J_{1} \leq 0.73$ and $0.315 \leq-J_{2} \leq 0.615$ and the corresponding critical temperatures were derived as described in detail in Secs. II and III For all $\left|J_{1}\right|<\left|J_{2}\right|$, the MC calculations yield AFM II ordering, whereas FM ordering results for all $\left|J_{1}\right|>\left|J_{2}\right|$. Figures 4(a) and (b) show the calculated $T_{N}$ and $T_{C}$ values (open symbols) as a function of $J_{1}$ and $J_{2}$, respectively. Since we are interested in the hydrostatic pressure effect, the smallest values of $J_{1}$ and $J_{2}$ were chosen close to the exchange parameters of bulk EuTe at ambient pressure (filled sym- bols) and the maximum values correspond to hydrostatic pressures up to about $17 \mathrm{GPa}$.

As shown by Fig. 4 , the Monte Carlo calculations yield a strongly nonlinear dependence of $T_{N}$ on $J_{1}$. Hence, the calculated data points were approximated by second degree polynomials for $T_{N}\left(J_{1}, J_{2}\right)$ and $T_{C}\left(J_{1}, J_{2}\right)$ of the general form

$$
T\left(J_{1}, J_{2}\right)=A+B J_{1}+C J_{2}+D J_{1}^{2}+E J_{1} J_{2}+F J_{2}^{2} .
$$

These approximations fit the Monte Carlo data with better than $\pm 0.1 \mathrm{~K}$ accuracy and are represented as solid lines in Figs. 4(a) and (b).

Using the normal pressure exchange constants $J_{1,0}=$ $0.192 \mathrm{~K}$ and $J_{2,0}=-0.313 \mathrm{~K}$ determined in Sec. III] the two branches of experimental data sets for the antiferromagnetic $T_{N}(a)$ and ferromagnetic $T_{C}(a)$ of Fig. 3 were fitted using Eqns. (18) and (19) with common exponents $n_{1}$ and $n_{2}$ and $r_{1}=a / \sqrt{(2)}$ and $r_{2}=a$. All experimental data points are weighted equally in the least square fit routine, which was performed on a logarithmic scale since equal weights may cause one branch to dominate if there is a difference in the magnitude of the dependent variable.

From this modeling, a Grüneisen exponent of $n_{1}=$ $20.6 \pm 0.4$ for the NN exchange $J_{1}$ and of $n_{2}=10.4 \pm 0.5$ for the NNN exchange $J_{2}$ were obtained. As demonstrated by the solid lines in Fig. 3. with these parameters the whole body of experimental findings, including the approximately constant Néel temperature $T_{N}$ at small hydrostatic strain, the transition from antiferromagnetic to ferromagnetic ordering at $a=6.29 \AA$ and the steep superlinear increase of the Curie temperature at high pressures, i.e., small lattice constants, are exactly reproduced. Moreover, the obtained power law exponent $n_{2}$ for $J_{2}\left(r_{2}\right)$ is in excellent agreement with Bloch's 57 10/3 law for the volume dependence of superexchange.

The such obtained dependence of the NN and NNN exchange integrals $J_{1}(a)$ and $J_{2}(a)$ as a function of lattice parameter $a$ is presented in Fig. 3 as dashed and dash-dotted line, respectively. Evidently, both exchange constants strongly increase with decreasing lattice constant. However, $J_{1}(a)$ increases much more rapidly than $J_{2}(a)$ due to the two times larger power law exponent. Therefore, the two curves intersect at $a=6.29 \AA$, where $J_{1} / k_{B}=-J_{2} / k_{B}=0.51 \mathrm{~K}$, and at smaller $a$, the ferromagnetic $J_{1}$ becomes the dominating exchange mechanism. For $f c c$ lattices with competing ferromagnetic NN exchange and antiferromagnetic NNN exchange interactions, this is exactly the condition for the material to become ferromagnetic ${ }^{5}$.

Our results are in severe contrast to the previous mean field analysis of Goncharenko et al. $\underline{46}$, who concluded from the negligible variation of $T_{N}$ in the antiferromagnetic state with changing lattice constant that the NNN exchange $J_{2}$ in $\mathrm{Eu} X$ should not depend on the lattice parameter. Consequently, the whole variation of $T_{C}(a)$ was attributed solely to changes in $J_{1}(a)$ using the mean 

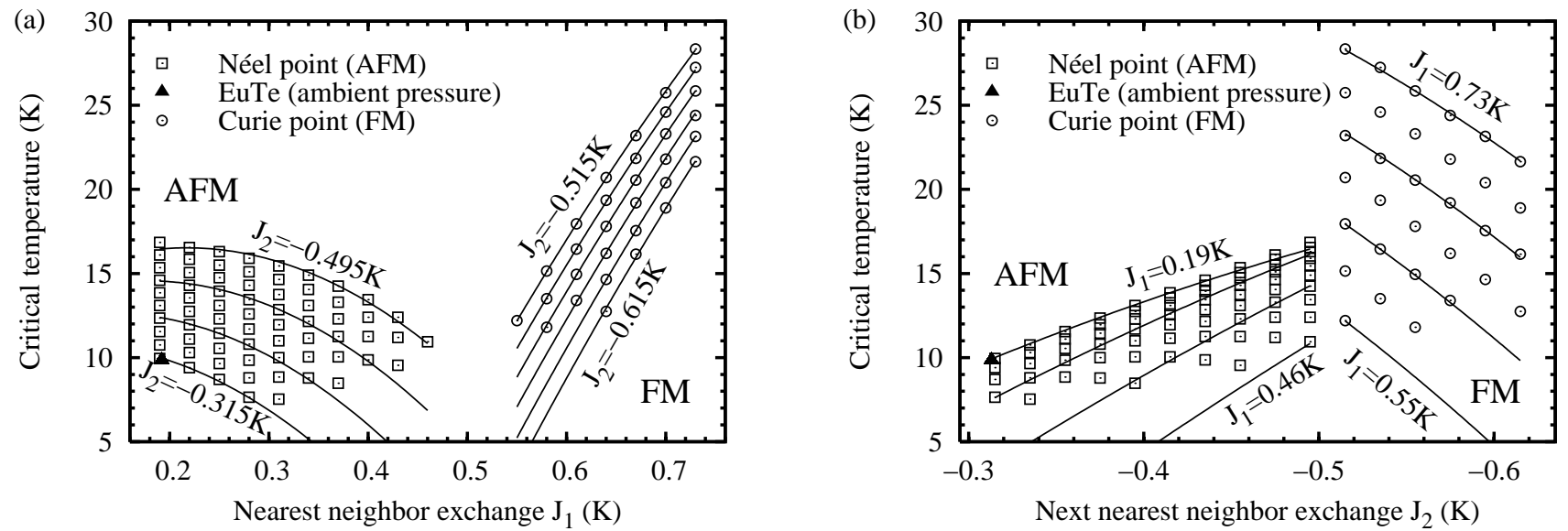

FIG. 4: Monte Carlo calculated critical temperatures of a bulk fcc Heisenberg system as functions of the strength of (a) the nearest neighbor exchange interaction $J_{1}$ and (b) the next-nearest neighbor exchange interaction $J_{2}$, showing antiferromagnetic to paramagnetic transitions (squares) when $J_{1}<\left|J_{2}\right|$ and ferromagnetic to paramagnetic transitions (circles) when $J_{1}>\left|J_{2}\right|$. Solid lines represent polynomial fit functions, which are quadratic in $J_{1}$ and $J_{2}$.

field expression for the Curie temperature of

$$
T_{C}^{\mathrm{MFA}}=\frac{2}{3} S(S+1)\left(12 J_{1}+6 J_{2}\right) / k_{B}
$$

with constant $J_{2}$ for data analysis. On the contrary, our calculation show that the broad plateau of $T_{N}(a)$ for lattice constants around $a=6.42 \AA$ just results from the fact that in the antiferromagnetic phase the ferromagnetic exchange drops faster than the antiferromagnetic exchange as the lattice constant increases.

\section{EXCHANGE INTERACTIONS IN EUO AND EuS}

As shown in the previous sections, the exchange constants obtained by Monte Carlo calculations strongly differ from previously published values. Therefore, to evaluate the distance dependence of the exchange constants of $\mathrm{EuO}$ and EuS, first the bulk values under ambient pressure have to be reexamined by the Monte Carlo method.

\section{A. Exchange constants at ambient pressure}

$\mathrm{EuO}$ and $\mathrm{EuS}$ are low temperature ferromagnets with Curie temperatures $T_{C}$ of $69.15 \mathrm{~K}^{74}$ and $16.6 \mathrm{~K}^{74.75}$, respectively. The magnetic properties are determined mainly by the dominant ferromagnetic NN exchange interaction $J_{1}$ in both materials. Compared to the case of antiferromagnetic EuTe, where only the Néel temperature and the critical field at $T=0 \mathrm{~K}$ are needed to deduce the exchange constants, the determination of $J_{1}$ and $J_{2}$ for ferromagnetic $\mathrm{EuO}$ and EuS is much more involved. As a result, there exists a substantial variation in the reported exchange constants for bulk $\mathrm{EuO}$ and $\mathrm{EuS}$ deduced from different experimental techniques like inelastic neutron scattering ${ }^{74,75,76}$, specific heat ${ }^{77,78,79,80}$, nuclear magnetic resonance $81.82,83$ (NMR) and spin wave resonance measurements $\underline{84}$ (see, e.g., Passell et al $\stackrel{74}{\text { for }}$ a review). Especially the values for the NNN exchange constant $J_{2}$ in $\mathrm{EuO}$ and $\mathrm{EuS}$ differ by up to a factor of two in literature and whether $J_{2}$ is ferromagnetic or antiferromagnetic in $\mathrm{EuO}$ is still a matter of debate. The most recent results based on inelastic neutron scattering studies on single crystals of $\mathrm{EuO}^{76}$ and $\mathrm{EuS}^{75}$ yielded $\left(J_{1,0}^{\mathrm{EuO}} / k_{B}, J_{2,0}^{\mathrm{EuO}} / k_{B}\right)=(0.625 \mathrm{~K}, 0.125 \mathrm{~K})$ and $\left(J_{1,0}^{\mathrm{EuS}} / k_{B}, J_{2,0}^{\mathrm{EuS}} / k_{B}\right)=(0.221 \mathrm{~K},-0.100 \mathrm{~K})$, respectively, consistent with Passell et al.' $s \underline{74}$ analysis on powdered samples. Notably, a ferromagnetic NNN exchange interaction was obtained for $\mathrm{EuO}$.

In all studies, the sum of $J=J_{1}+J_{2}$ has been more reliably determined than the individual $\mathrm{NN}$ and NNN exchange interactions, and this sum is quite consistent among the various studies. For EuO single crystals, $J^{\mathrm{EuO}} / k_{B}=0.755 \mathrm{~K}$ was obtained by Comment et al $\stackrel{83}{\text { s. }}$ from NMR measurements, in agreement with neutron scattering studies by Mook at al. $\stackrel{76}{ }$, and this value also agrees with the results obtained by neutron scattering ${ }^{74}$ and specific heat measurements 80 on powdered samples. A very good agreement for $J_{1}+J_{2}$ also exist among respective studies for $\operatorname{EuS} 74,75,80,81$, from which we calculate $J / k_{B}^{\mathrm{EuS}}=0.121 \pm 0.003 \mathrm{~K}$ as mean value.

To determine the exchange constants by the Monte Carlo method, we again performed a series of calculations for the model Heisenberg Hamiltonian of Eqn. (1) with the NN and NNN exchange interactions $J_{1}$ and $J_{2}$ varied independently over a wide range of $0.5 \leq J_{1} \leq 2.5$ and $0.4 \leq-J_{2} \leq 0.8$. We find ferromagnetic ordering for all combinations of $J_{1}>-J_{2}$ and determined the corresponding critical ordering temperature as a function of $J_{1}$ and $J_{2}$ as presented in Fig. 5 (a) and (b), respectively. 
Evidently, $T_{C}$ increases linearly with increasing NN exchange constant $J_{1}$, but decrease when $J_{2}$ increases. As shown by the solid lines in Fig. 5 , the general dependence of $T_{C}$ on the exchange constants can be well described by the relation

$$
\begin{aligned}
T_{C}^{\mathrm{MC}} & =79.0 J_{1} / k_{B}+55.9 J_{2} / k_{B} \\
& =0.627 \frac{2}{3} S(S+1) 12\left(J_{1}+0.708 J_{2}\right) / k_{B},
\end{aligned}
$$

which differs considerably compared to the mean field expression of Eqn. (20). Yet, the critical coupling $J_{1} /\left(k_{B} T_{C}\right)$ closely resembles the theoretical predictions from high-temperature series expansion ${ }^{60}$. Inserting the $\mathrm{EuO}$ and EuS exchange values of $\mathrm{Mook}^{76}$ and Bohn et al $\frac{75}{7}$ in our Monte Carlo relation of Eqn. (21) yields Curie temperatures of only $56 \mathrm{~K}$ and $12 \mathrm{~K}$, respectively, which is much lower than the measured experimental values. This shows that, like for EuTe, the exchange constants have been considerably underestimated in both materials.

Using the experimental values for $J=J_{1}+J_{2}$ quoted above, the critical Curie temperature $T_{C}$ can be calculated as a function of the exchange constant $J_{1}$ using Eqn. (21) and $J_{2}=J-J_{1}$. The results are plotted as solid lines in Fig. [6(a) and (b) for $\mathrm{EuO}\left(J^{\mathrm{EuO}}=0.755 \mathrm{~K}\right)$ and $\operatorname{EuS}\left(J^{\mathrm{EuS}}=0.121 \mathrm{~K}\right)$, respectively. From the intersection of these lines with the respective experimental $T_{C}$ values of $69.15 \mathrm{~K}^{74}$ and $16.6 \mathrm{~K}^{74.75}$ (horizontal dashed lines in Fig. (6), the bulk exchange constants of $J_{1}^{\mathrm{EuO}}=1.169 \mathrm{~K}$ and $J_{2}^{\mathrm{EuO}}=-0.414 \mathrm{~K}$ are obtained for $\mathrm{EuO}$ and of $J_{1}^{\mathrm{EuS}}=0.427 \mathrm{~K}$ and $J_{2}^{\mathrm{EuS}}=-0.306 \mathrm{~K}$ for EuS. In Fig. 6, also plotted are the $T_{C}$ values expected from the mean field approximation (dash-dotted line, Eqn. (201) as well as from a series expansion es-

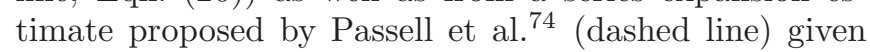
by

$$
T_{C}^{\mathrm{SE}, \text { est. }}=0.790 \frac{2}{3} S(S+1) 12\left(J_{1}+0.619 J_{2}\right) / k_{B} .
$$

Evidently, in both cases, much higher critical temperatures are predicted for a given set of exchange constants, i.e., from the observed transition temperatures, the exchange integrals are strongly underestimated. Moreover, a ferromagnetic NNN exchange would be suggested for $\mathrm{EuO}$. Our results exclude such a ferromagnetic NNN exchange, i.e., a negative $J_{2}$ is obtained for $\mathrm{EuO}$ even if $J^{\mathrm{EuO}}$ is underestimated by as much as $15 \%$. Thus, as already found for the EuTe case, our Monte Carlo calculations greatly revise the bulk exchange constants.

\section{B. Distance dependence of EuO and EuS exchange constants}

For $\mathrm{EuO}$ and $\mathrm{EuS}$, the Curie temperature $T_{C}$ strongly increases with increasing hydrostatic pressure, i.e., decreasing lattice constant. This is illustrated by Figs. 7 and 8 , where the experimentally determined $T_{C}$ values of
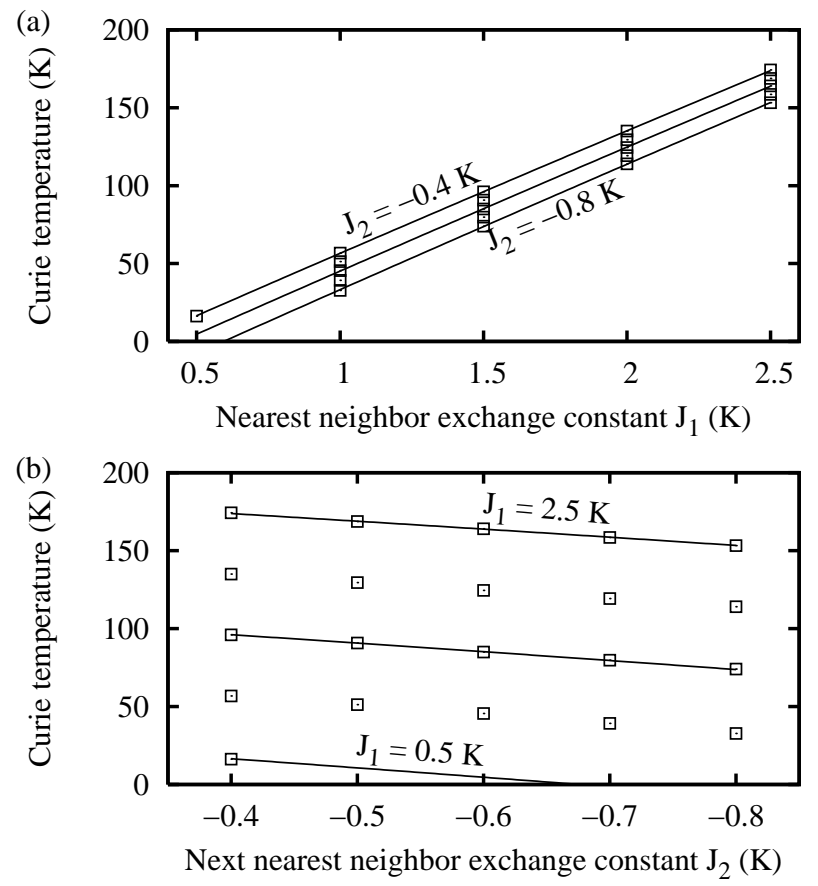

FIG. 5: Monte Carlo calculated critical temperatures of a bulk $f c c$ Heisenberg system as functions of the strength of (a) the nearest and (b) next-nearest neighbor exchange interactions ( $J_{1}$ and $J_{2}$, respectively), showing ferromagnetic to paramagnetic transitions if $J_{1}>\left|J_{2}\right|$. The parameter range covers $\mathrm{EuO}$ and EuS under hydrostatic pressures between 0 and $20 \mathrm{GPa}$. Solid lines represent fit functions, which are linear in $J_{1}$ and $J_{2}$-see Eqn. (21).

$\mathrm{EuO}^{35,44,45}$ and $\mathrm{EuS} 48$ are plotted as a function of the lattice constant. At hydrostatic pressures around $20 \mathrm{GPa}$, corresponding to a $6-8 \%$ reduction of the lattice constant, $T_{C}$ is as high as $200 \mathrm{~K}^{45}$ and $180 \mathrm{~K}^{48}$, respectively. For $\mathrm{EuO}$, the experimental data $T_{C}(a)$ of Fig. 7 is compiled from three independent investigations $35.44,45$, with McWhan et al.'s data combined with the pressure-volume relation taken from Ref. 85. For EuS, the data is taken from Ref. 48. For EuO, experiments at even higher hydrostatic pressures up to $31 \mathrm{GPa}^{45}$ have revealed that the ferromagnetic ground state becomes unstable at around $23 \mathrm{GPa}$ and that the Curie temperature drops sharply afterwards instead of further increasing. In Ref. 45 this behavior was attributed to $s f$ hybridization competing with $s f$ exchange in this pressure range. Therefore, we restrict our analysis to the $0-20 \mathrm{GPa}$ range, i.e., lattice constants above $4.9 \AA$, where such effects seem not to be of importance.

To determine the interatomic distance dependence of the exchange constants, we proceed in the same manner as described in Sec. IV by fitting the calculated $T_{C}^{\mathrm{MC}}\left(J_{1}, J_{2}\right)$ dependence of Eqn. (21) obtained by the Monte Carlo calculations to the data of the hydrostatic pressure experiments, applying the magnetic Grüneisen law (Eqn. (18)) as functional dependence for the NN and NNN exchange constants. As input parameters we use 

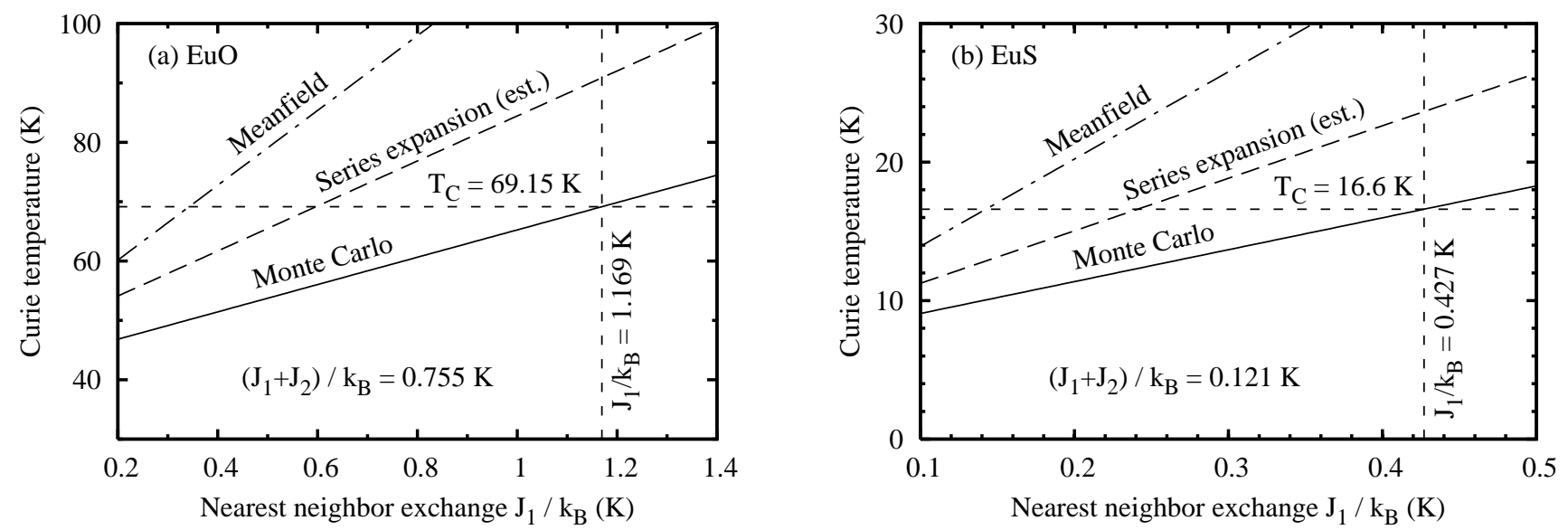

FIG. 6: Determination of the intrinsic nearest neighbor exchange constant $J_{1,0}$ in (a) EuO and (b) EuS from Monte Carlo results (solid lines) under the constraint $J=J_{1}+J_{2}=$ const.. Experimental values for $J$ are taken from Refs. 83 (EuO) and 75 (EuS). The Curie temperature as functions of $J_{1}$ as derived from mean field approximation (dash-dotted line) and an approximate series expansion results ${ }^{74}$ (dashed line) are shown in comparison. The dashed horizontal lines indicate the measured bulk $T_{C}$ values of 69.15 and $16.6 \mathrm{~K}$ for $\mathrm{EuO}$ and $\mathrm{EuS}$, respectively.

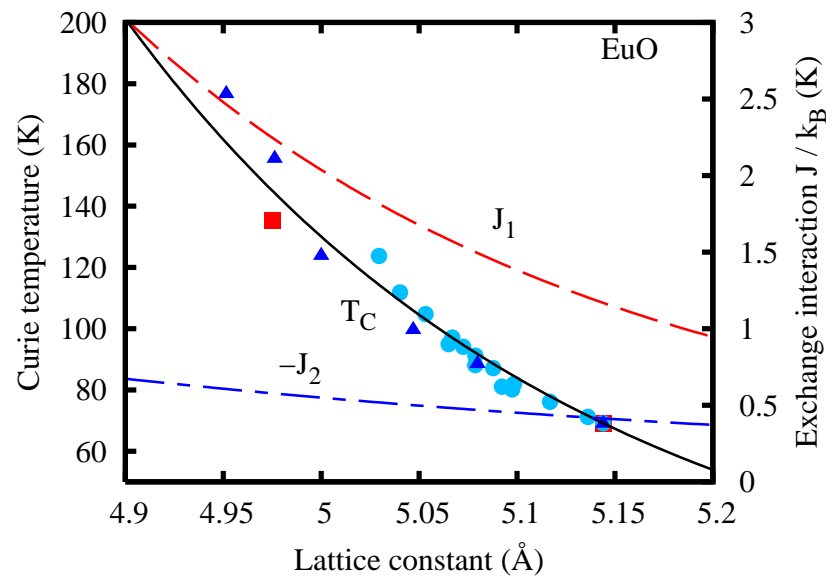

FIG. 7: (color online) Critical temperatures and exchange interactions in $\mathrm{EuO}$ as functions of the lattice parameter; filled symbols ( circles $^{35}$, triangles ${ }^{44}$, squares ${ }^{45}$ ) correspond to experimental Curie points; the solid line represents the least square fit of Monte Carlo critical temperatures $T_{C}\left(J_{1}, J_{2}\right)$ based on the magnetic Grüneisen law $J_{i}\left(r_{i}\right) \sim r_{i}^{-n_{i}}$. The corresponding dependence of the nearest $\left(J_{1}\right)$ and next-nearest neighbor exchange interaction $\left(J_{2}\right)$ on the lattice parameter is plotted as the dashed and dash-dotted line, respectively.

the bulk exchange constants $J_{1,0}^{\mathrm{MC}}$ and $J_{2,0}^{\mathrm{MC}}$ determined in the previous section and treat the power law exponents $n_{1}$ and $n_{2}$ in Eqn. (18) as adjustable parameters. It turns out, that because the $\mathrm{NN}$ exchange $J_{1}$ in $\mathrm{EuO}$ and $\mathrm{EuS}$ is always much larger than the NNN exchange $J_{2}$, the $T_{C}(a)$ dependence is quite insensitive to the variation of $J_{2}$ as a function of lattice constant, i.e., the fit yields only unreliable values for $n_{2}$. Because for EuTe we have already confirmed Bloch's 10/3 law for the volume dependence of the NNN superexchange integral $J_{2}$, we have therefore cho-

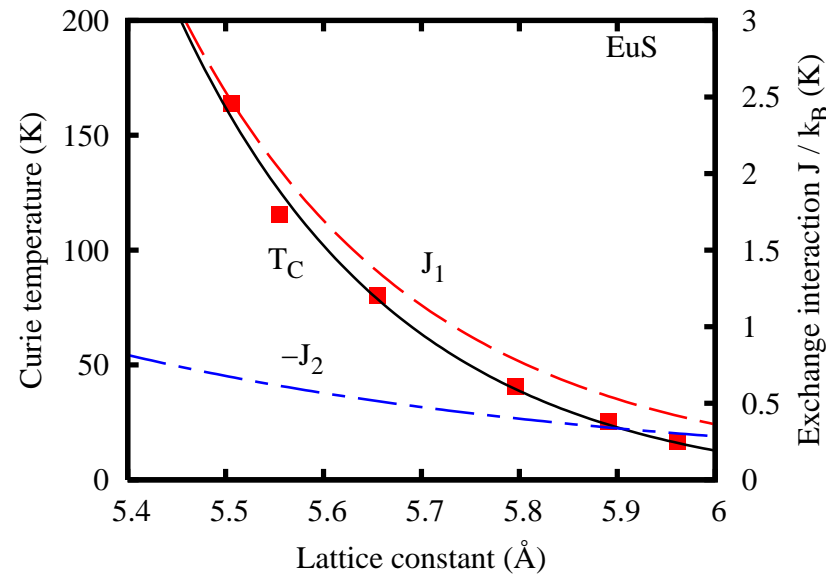

FIG. 8: (color online) Critical temperatures and exchange interactions in EuS as functions of the lattice parameter; squares $\frac{48}{}$ correspond to experimental Curie points; the solid line represents the least square fit of Monte Carlo critical temperatures $T_{C}\left(J_{1}, J_{2}\right)$ based on the magnetic Grüneisen law $J_{i}\left(r_{i}\right) \sim r_{i}^{-n_{i}}$. The corresponding dependence of the nearest $\left(J_{1}\right)$ and next-nearest neighbor exchange interaction $\left(J_{2}\right)$ on the lattice parameter is plotted as the dashed and dash-dotted line, respectively.

sen to fix the distance dependence of $J_{2}(a)$ proportional to $r_{2}^{-10}$ for EuO and EuS as well. From the fit, we then obtain $n_{1}^{\mathrm{EuO}}=19.6 \pm 0.4$ and $n_{1}^{\mathrm{EuS}}=22.4 \pm 0.3$ as the Grüneisen exponents for the NN exchange interaction. The resulting lattice constant dependence of the Curie temperatures $T_{C}(a)$ and exchange interactions $J_{1}(a)$ and $J_{2}(a)$ are plotted in in Figs. 7 for $\mathrm{EuO}$ and 8 for $\mathrm{EuS}$ as solid, dashed and dash-dotted lines, respectively. Evidently, an excellent fit with the experimental data is obtained over the whole lattice parameter range for both 


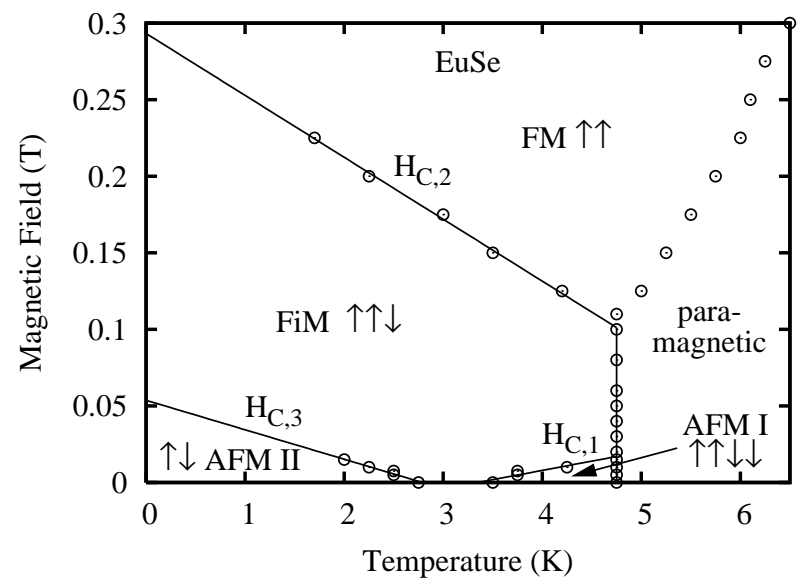

FIG. 9: Experimental magnetic phase diagram of EuSe. The phase boundaries (solid lines) are obtained from a linear least square fit to experimental data (open symbols). See Ref. 11 for details.

materials. This is also an indication that the choice of $n_{2}=10$ is a reasonable assumption. It is also noted that due to the about a factor of two larger Grüneisen exponent of $J_{1}$ compared to that of $J_{2}$, at high hydrostatic pressures (small lattice constants), the NN exchange $J_{1}$ is as much as five times larger than the NNN exchange $J_{2}$. Thus, the ferromagnetic NN exchange completely dominates the magnetic behavior of both materials.

\section{EXCHANGE INTERACTION IN EuSe}

Unlike EuO, EuS and EuTe, which exhibit stable magnetic low temperature phases, EuSe is a metamagnet with at least four different known ordered magnetic phases, i.e., two antiferromagnetic phases of type I (AFM I) and type II (AFM II), a ferrimagnetic phase (FiM) and a ferromagnetic phase (FM). Figure 9 shows the $H-T$ phase diagram of unstrained EuSe derived from susceptibility measurements on several micrometer thick epitaxial layers ${ }^{11}$, and the corresponding different spin configurations are illustrated by the arrows. The phase boundaries shown in Fig. 9 are in good agreement with earlier publications ${ }^{9,10}$. In particular, the AFM I Néel point of $T_{N, \mathrm{AFM} \mathrm{I}}=4.7 \mathrm{~K}$ and the critical field of $H_{C, 3}(0)=0.05 \mathrm{~T}$ for the transition from AFM II to FiM obtained by linear extrapolation of the experimental AFM II to FiM phase boundary are in excellent agreement with Refs. 9 and 10 .

The metamagnetic behavior of EuSe at ambient pressure shows similarities to the situation in EuTe at the AFM II to FM transition that occurs at a hydrostatic pressure of $9 \mathrm{GPa}$ (see Fig. 3), where $J_{1} \approx\left|J_{2}\right|$. The appearance of an AFM I phase at this pressure that accompanies the AFM II to FM phase transition ${ }^{46}$ shows that at this pressure EuTe is metamagnetic too. Thus, in both materials a metamagnetic behavior occurs when $J_{1}$

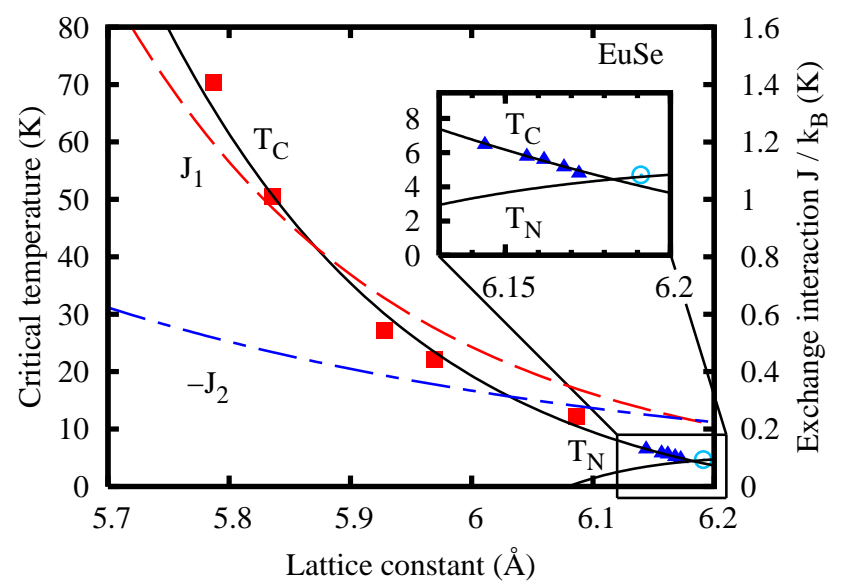

FIG. 10: (color online) Critical temperatures and exchange interactions in EuSe as functions of the lattice parameter; filled symbols - triangles ${ }^{40}$ and squares ${ }^{48}$ correspond to experimental Curie points. The solid line $\left(T_{C}\right)$ represents the least square fit of the Monte Carlo $T_{C}\left(J_{1}, J_{2}\right)$ to the experimental Curie points based on the magnetic Grüneisen law $J_{i}\left(r_{i}\right) \sim r_{i}^{-n_{i}}$. The corresponding nearest and next-nearest neighbor exchange interaction, $J_{1}$ and $J_{2}$ as functions of the lattice parameter $a$, are represented as the dashed and dashdotted lines, respectively. The inset shows the AFM I-FM transition occurring in EuSe at hydrostatic pressures around $0.5 \mathrm{GPa}$. The solid graph $\left(T_{N}\right)$, which is based on an extrapolation of $J_{1}(a)$ and $J_{2}(a)$ towards $a_{0}$ corresponds to a theoretical AFM II Néel temperature which is energetically compareable to the experimentally observed AFM I Néel point (open symbol ${ }^{11,40}$ ).

and $\left|J_{2}\right|$ are approximately equal and cancel each other. Then otherwise negligible additional interactions come into play. As shown by Fig. 10, when applying hydrostatic pressure of $0.5 \mathrm{GPa}^{40}$ EuSe becomes a stable ferromagnet and at increasing pressures up to $15 \mathrm{GPa}$, i.e., $6 \%$ reduction of the lattice constant, the ferromagnetic ordering temperature increases from 4.7 to above $70 \mathrm{~K}^{48}$. The corresponding experimental $T_{C}(a)$ data of EuSe is displayed as filled symbols in Fig. 10. Lechner et al. ${ }^{11}$ also showed that the introduction of only little biaxial strain in EuSe drastically expands the boundaries of the AFM II phase and causes the AFM I phase to disappear completely. Thus, an AFM II to paramagnetic Néel point $T_{N \text {,AFM II }}$ is observed in strained EuSe, which is again similar to the situation in EuTe.

The magnetic phase diagram of EuSe and its metastability for already small lattice deformations ${ }^{11}$ cannot be described by isotropic NN and NNN Heisenberg exchange interactions alone. Especially, the ferrimagnetic and the AFM I phases, which show a magnetic structure with a periodicity of three, respectively, four atomic layers require further distant exchange interactions and/or other types of magnetic interactions such as long-range dipolar interactions ${ }^{10}$. Moreover, using MFA and a Hamiltonian, which includes the exchange interaction up to the third nearest neighbor $\left(J_{3}\right)$ and dipolar interactions Fukuma 
et al $\stackrel{10}{n}$ showed that the critical field $H_{C, 3}$ at $T=0$ is independent of $J_{3}$ and depends only on the sum $\left(J_{1}+J_{2}\right)$ as well as the dipolar coupling strength $D$. A good esti- mate of $\left(J_{1}+J_{2}\right)$ can be calculated using the mean field relation

$$
2\left(J_{1,0}+J_{2,0}\right)=-\frac{1}{3 S} g \mu_{B} H_{C, 3}(0)-\left[D_{x x}\left(\boldsymbol{Q}_{\boldsymbol{L}}\right)-\frac{1}{9} D_{x x}(\mathbf{0})-\frac{8}{9} D_{x x}\left(\frac{2}{3} \boldsymbol{Q}_{\boldsymbol{L}}\right)\right] .
$$

Here $D_{x x}\left(\boldsymbol{Q}_{\boldsymbol{L}}\right), D_{x x}(\mathbf{0})$ and $D_{x x}\left(2 \boldsymbol{Q}_{\boldsymbol{L}} / 3\right)$ correspond to the dipole coupling strength for spins lying in the (111) plane (see Ref. 10 for exact definitions). Inserting $H_{C, 3}(0)=0.05 \mathrm{~T}$ and the calculated values of $D_{x} x$ given in Ref. 10 in Eqn. (23), we obtained $\left(J_{1}+J_{2}\right) / k_{B}=$ $-5.2 \mathrm{mK}$. Unlike for the other $\mathrm{Eu} X$ compounds, we were not able to derive any further reliable condition that would allow to determine $J_{1}$ and $J_{2}$ independently of the high pressure data in EuSe.

Since our Heisenberg spin model can only generate FM and AFM II orderings, we are only able to reproduce the behavior of $T_{C}$ as a function of lattice constant in the strain-induced ferromagnetic phase of EuSe at pressures above $0.5 \mathrm{GPa}$. In this region, third nearest neighbor exchange and dipolar interactions are not expected to contribute significantly to the ferromagnetic ordering. Therefore, we calculated the ferromagnetic ordering temperature as functions of the NN and NNN Heisenberg exchange interaction in the range $0.2 \mathrm{~K} \leq J_{1} \leq 1.0 \mathrm{~K}$ and $-0.175 \mathrm{~K} \geq J_{2} \geq-0.4 \mathrm{~K}$ with $J_{1}>\left|J_{2}\right|$. As shown in Fig. 11] $T_{C}\left(J_{1}, J_{2}\right)$ is slightly nonlinear in both $J_{1}$ and $J_{2}$, but approaches the relation given in Eqn. (21) for $J_{1} \gg-J_{2}$. We also simulated the AFM II to paramagnetic transitions for $J_{1}<\left|J_{2}\right|$ in the interval $0.13 \mathrm{~K} \leq$ $J_{1} \leq 0.18 \mathrm{~K}$ and $-0.15 \mathrm{~K} \geq J_{2} \geq-0.2 \mathrm{~K}$. The calculated critical temperature $T_{N}$ as a function of the exchange constants $J_{1}$ and $J_{2}$ are shown in Figs. 11(a) and (b) as open squares. As in the case of EuTe, $T_{N}\left(J_{1}, J_{2}\right)$ is strongly nonlinear and both $T_{C}\left(J_{1}, J_{2}\right)$ and $T_{N}\left(J_{1}, J_{2}\right)$ were approximated by second order polynomials as given in Eqn. (19).

Inserting the magnetic Grüneisen law of Eqn. (18) $J_{1}\left(r_{1}\right)=J_{1}(a / \sqrt{2})$ and $J_{2}\left(r_{2}\right)=J_{2}(a)$ into the obtained $T_{C}\left(J_{1}, J_{2}\right)$ dependence, the distance dependence of the EuSe exchange constants $J_{i}(a)$ was again obtained by fitting the calculated $T_{C}(a)$ to the experimental Curie points of EuSe under hydrostatic pressure (filled symbols). Other than in the preceding sections, not only the Grüneisen exponents $n_{1}$ and $n_{2}$ but also the ambient pressure exchange constants $J_{1,0}$ and $J_{2,0}$ were used as adjustable parameters in the fit routine, only restricted by the condition $\left(J_{1,0}+J_{2,0}\right) / k_{B}=-5.2 \mathrm{mK}$, obtained from the critical field $H_{C, 3}(0)$ as described above. Unlike the situation in $\mathrm{EuO}$ and $\mathrm{EuS}$, the bahavior of $J_{2}(a)$ influences the magnetic ordering considerably in the region close to $a_{0}$. Eventually, $J_{1,0} / k_{B}=0.223 \pm 0.016 \mathrm{~K}$,
$J_{2,0} / k_{B}=-0.228 \pm 0.016 \mathrm{~K}, n_{1}=24.9 \pm 1.8$ and $n_{2}=$ $12.2 \pm 6.0$ are obtained by the fit. As is demonstrated by the solid line in Fig. 10, with these parameters the experimental $T_{C}(a)$ data are precisely reproduced. The dependence of $J_{1}$ and $J_{2}$ versus lattice constant are depicted as dashed, respectively, dash-dotted lines in Fig. 10, At the bulk EuSe lattice constant of $a_{0}=6.191 \AA$, the calculated $J_{2}$ is slightly larger in absolute value than $J_{1}$. This changes drastically as the lattice constant is reduced under hydrostatic pressure, with $J_{1}$ crossing $J_{2}$ already at low hydrostatic strain and $J_{1}$ becoming the dominant exchange interaction for $a<6.15 \AA$. The Grüneisen exponents for the NN and the NNN exchange interaction are again in good agreement with the results obtained for EuTe, EuO and EuS.

To further justify our results on EuSe, we substituted the obtained $J_{1}(a)$ and $J_{2}(a)$ into the theoretical $T_{N}\left(J_{1}, J_{2}\right)$ AFM II to paramagnetic Néel function, obtained from the fit of Eqn. (19) to the squares in Fig. 11] Extrapolating $T_{N}\left(J_{1}(a), J_{2}(a)\right)$ to the bulk lattice constant $a_{0}=6.191 \AA$ of EuSe, we obtained $T_{N, \text { AFM II }}^{\mathrm{MC}}\left(a_{0}\right)=4.5 \mathrm{~K}$, which is, as expected, above the experimentally observed AFM II to FiM transition temperature of around $2 \mathrm{~K}^{9,11}$, but below the AFM I Néel point of $4.7 \pm 0.1 \mathrm{~K}^{9,11,40}$ (open symbol in Fig. 10). That the calculated AFM II to paramagnetic transition temperature of $T_{N, \mathrm{AFM} \mathrm{II}}^{\mathrm{MC}}\left(a_{0}\right)$ is very close to the experimentally observed AFM I to paramagnetic $T_{N \text {, AFM I }}$ is also expected in mean field theory, where

$$
\begin{aligned}
T_{N, \mathrm{AFM} \mathrm{I}}^{\mathrm{MFA}}\left(J_{1}, J_{2}\right) & =4 S(S+1) J_{1} \\
T_{N, \mathrm{AFM} \mathrm{II}}^{\mathrm{MFA}}\left(J_{1}, J_{2}\right) & =-4 S(S+1) J_{2} .
\end{aligned}
$$

Thus, $T_{N, \text { AFM I }}$ and $T_{N \text {,AFM II }}$ are nearly equal when $J_{1}$ and $J_{2}$ are almost equal in strength. Moreover, it can easily be shown, that taking third-nearest neighbor or biquadratic exchange terms into account would not favor either of the two antiferromagnetic ordering types (at least in the mean field approximation) and the influence of dipolar interactions on the ordering temperatures is typically of the order of less than one Kelvin (see, e.g., chap. 4 of Ref. 86). In addition, Lechner et al.'s 11 results on biaxially strained EuSe indicate, that the AFM I ordering observed in unstrained EuSe with $T_{N, \text { AFM I }} \approx 4.7 \mathrm{~K}$ is energetically only slightly lower than the Néel point of the AFM II ordering and small biaxial strain produces a transition from one to the other. 

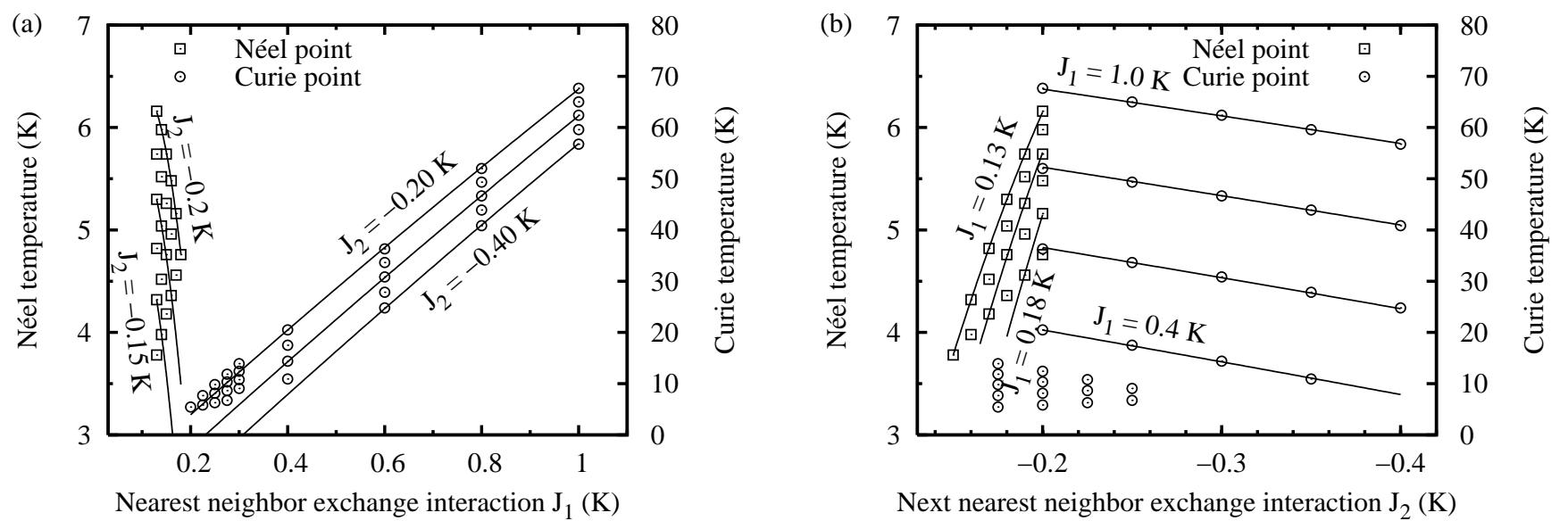

FIG. 11: Monte Carlo calculated critical temperatures of a bulk fcc Heisenberg system as a function of the strength of (a) the nearest and (b) next nearest neighbor exchange interactions $J_{1}$ and $J_{2}$, respectively, showing ferromagnetic to paramagnetic transitions if $J_{1}>\left|J_{2}\right|$ and antiferromagnetic to paramagnetic transitions if $J_{1}<\left|J_{2}\right|$. The parameter range corresponds to EuSe under hydrostatic pressures between 0 and around $15 \mathrm{GPa}$. Solid lines represent polynomial fit functions, which are quadratic in $J_{1}$ and $J_{2}$.

\begin{tabular}{lcccc}
\hline \hline & EuTe & EuSe & EuS & EuO \\
\hline$a_{0}(\AA)$ & 6.598 & 6.191 & 5.956 & 5.144 \\
$T_{N}\left(a_{0}\right)(\mathrm{K})$ & 9.85 & 4.7 & - & - \\
$T_{C}\left(a_{0}\right)(\mathrm{K})$ & - & - & 16.6 & 69.15 \\
\hline$J_{1,0}(\mathrm{~K})$ & 0.192 & 0.223 & 0.427 & 1.169 \\
$n_{1}$ & 20.6 & 24.9 & 22.4 & 19.6 \\
$\alpha_{1}\left(\AA^{-1}\right)$ & 4.56 & 5.99 & 5.47 & 5.44 \\
\hline$J_{2,0}(\mathrm{~K})$ & -0.313 & -0.228 & -0.306 & -0.414 \\
$n_{2}$ & 10.4 & 12.2 & $\left.10^{*}\right)$ & $\left.10^{*}\right)$ \\
$\alpha_{2}\left(\AA^{-1}\right)$ & 1.63 & 2.31 & $1.68^{*)}$ & $1.94^{*}$ \\
\hline \hline
\end{tabular}

TABLE II: Distance dependence of nearest (subscript 1) and next-nearest neighbor (subscript 2) exchange interactions of europium chalcogenides under hydrostatic pressure as obtained in our Monte Carlo study. For hydrostatic strains of typically less than $\approx 10 \%$, the exchange interactions can equally well be described by either magnetic Grüneisen laws, $J_{i}=J_{i, 0}\left(r_{i} / r_{0, i}\right)^{-n_{i}}$ or by simple exponential laws, $J_{i}=J_{i, 0} \exp \left[-\alpha_{i}\left(r_{i}-r_{0, i}\right)\right]$ - values labelled by $\left.{ }^{*}\right)$ are assumptions.

\section{DISCUSSION}

The exchange constants of all four $\operatorname{Eu} X$ compounds and their dependence on the lattice parameter obtained by our MC analysis are summarized in Tab. П1 and Fig. 12. Evidently, in all cases, the bulk exchange constants $J_{1,0}$ and $J_{2,0}$ are almost a factor of two larger compared to the values reported in previous works, which in their analysis did not take spin fluctuations into account. More importantly, we have found that the magnetic properties and critical phase transition temperatures of all $\mathrm{Eu} X$ compounds as a function of hydro-

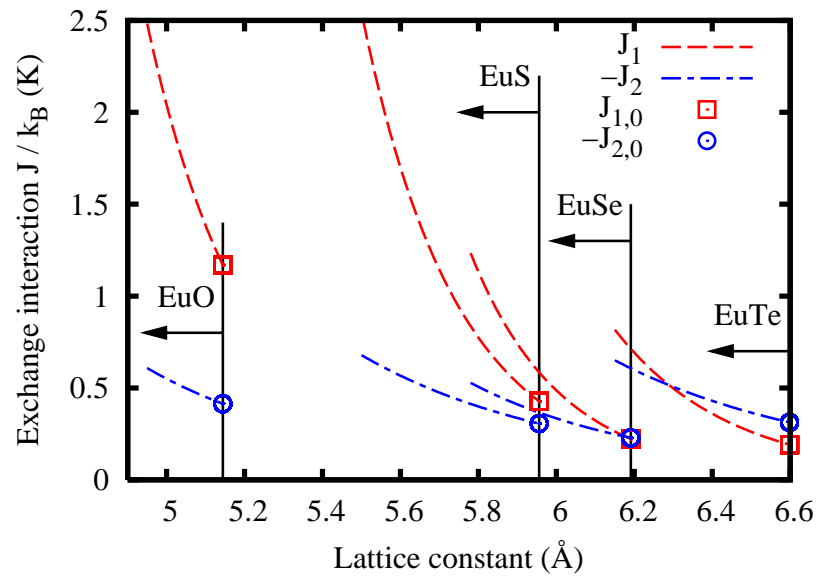

FIG. 12: (color online) Exchange interactions in europium chalcogenides as functions of the lattice parameter.

static strain, i.e., lattice parameter, can be consistently described by the magnetic Grüneisen power law dependence $J_{i}\left(r_{i}\right)=J_{i, 0}\left(r_{i} / r_{i, 0}\right)^{-n_{i}}$ with characteristic power law exponents of $n_{1} \approx 10$ for the NN exchange $J_{1}$ and of $n_{2} \approx 20$ for the NNN exchange $J_{2}$ as indicated in Tab. II.

The corresponding dependence of the exchange interactions on the lattice constant $J_{1}(a)$ and $J_{2}(a)$ for all compounds are represented as dashed lines in Fig. 12, Evidently, neither $J_{1}(a)$ nor $J_{2}(a)$ are continuous functions over different members of the $\operatorname{Eu} X$ family. Therefore, the effect of the substitution of the anion elements cannot be simplified to a variation in the lattice constant alone, as was already noted in the previous work of Goncharenko et $a l \stackrel{54}{\underline{5}}$. In fact, as shown by Fig. 12, for a fixed lattice constant the absolute values of the exchange constants $J_{1}$ and $J_{2}$ are always much larger for the com- 
pound with larger anion element, i.e.,

$$
\left|J_{i}^{\mathrm{EuTe}}(a)\right| \gg\left|J_{i}^{\mathrm{EuSe}}(a)\right| \gg\left|J_{i}^{\mathrm{EuS}}(a)\right| \gg\left|J_{i}^{\mathrm{EuO}}(a)\right|
$$

For this reason, at a given lattice parameter the ordering temperature is always significantly larger in EuTe compared to EuSe, EuS and EuO. This is consistent with the extrapolation of the $T_{C}(a)$ data obtained from experiments.

Apart from the empirical magnetic Grüneisen law of Eqn. (18) as the inter-ion distance dependence of the exchange interactions in the $\mathrm{Eu} X \mathrm{~s}$, we have also explored, if different functional dependencies might reproduce the experimental ordering temperatures as functions of the lattice constant as well. Due to the strong superlinear increase of the ferromagnetic ordering temperature with decreasing lattice constant and the fact that $T_{C}\left(J_{1}, J_{2}\right)$ behaves very close to linear in all of the $\mathrm{Eu} X$ compounds, a linear distance dependence of the exchange interactions can be categorically ruled out. One other empirical form of $J(r)$, which could be expected from the nature of quantum mechanical two-electron multicenter integrals, e.g., the Heitler-London approach to solve the Schrödinger equation for the hydrogen molecule ${ }^{87}$, is a simple exponential distance law of the form

$$
\begin{aligned}
J(r) & =J_{0} \exp \left[-\alpha\left(r-r_{0}\right)\right] \\
& =J_{0} \exp \left[-\alpha r_{0}\left(r / r_{0}-1\right)\right],
\end{aligned}
$$

where $J\left(r_{0}\right)=J_{0}$ is again the exchange constant under normal condition. Applying this exponential law in the simulated critical temperatures $T_{N}\left(J_{1}, J_{2}\right)$ and $T_{C}\left(J_{1}, J_{2}\right)$ and fitting the experimental critical points of the $\mathrm{Eu} X \mathrm{~s}$ as functions of the lattice constant, it turns out, that the results for $J_{1}(a)$ and $J_{2}(a)$ are practically indistinguishable from those received by exploring the Grüneisen power law. In essence, the fitted $T_{N}(a)$ and $T_{C}(a)$ curves and the $J_{1}(a)$ and $J_{2}(a)$ distance dependencies of the exchange interactions coincide almost exactly with those presented in Fig. 12 The reason for this unambiguity is that the variations in the interatomic distances achievable by hydrostatic strain are generally too small $(\leq 8 \%)$ to be able to definitely single out between the two functional dependencies. However, while the distance dependencies of the exchange interaction for the different members of the $\mathrm{Eu} X$ family show consistant Grüneisen exponents $n_{i}$ for the NN and the NNN exchange interactions, such similarities can not be expected for the scaling factors $\alpha_{i}$ in the simple exponential description. This follows from the fact that only the magnetic Grüneisen law of Eqn. (18) is defined in terms of relative changes of the lattice parameter $r / r_{0}$. This, however, can also be achieved by redefining the exponential distance law as a function of $r / r_{0}$ (Eqn. (28)). Then $\alpha r_{0}$ represents a dimensionless scaling factor that can be compared for the different members of the $\mathrm{Eu} X$ family. The calculated scaling factors $\alpha_{i}$ are given together with the $n_{i}$ and the $J_{0, i}$ in Tab. II and are in the range of 4.5$6 \AA^{-1}$ for $\alpha_{1}$ and $1.6-2.3 \AA^{-1}$ for $\alpha_{2}$. As it turns out, the values for $\alpha_{i} r_{0}$ are approximately equal to the respective $n_{i}$ s for all EuX compounds. This becomes clear from a more mathematical point of view. Using the relation ${ }^{88}$

$$
\alpha=-\frac{\frac{d J}{d r}(r)}{J(r)},
$$

and requiring that both $J(r)$ and its derivative with respect to $r$ have to match at $r_{0}$ yields the relation $n=\alpha r_{0}$. Thus, we have shown that for small deviations of $r$ from $r_{0}$ the power law and the exponential dependence are nearly equivalent when $\alpha$ is chosen as $\alpha=n / r_{0}$.

The theoretical derivation of analytic scaling laws for the distance dependence of the exchange interactions is far from trivial and estimates can only be obtained as far as the exchange mechanisms are understood. According to Kasuya ${ }^{4}$ the most important contribution to the NN exchange interaction consists of a virtual excitation of a $\mathrm{Eu}^{2+} 4 f$ electron to the $5 d$ state of a NN cation and a subsequent intra-atomic $d$ - $f$ exchange. For this type of exchange, a distance dependence $J_{1}\left(r_{1}\right) \sim \exp \left(-8 r_{1} / r_{0}\right)$ is considered. The NNN exchange may consist of several competing components, which are considered to involve excitations of the anion $p$ electrons to neighboring cation $5 d$ states. To our knowledge Kasuya did not give an estimate for the distance dependence of the NNN superexchange. Lee and Liu ${ }^{71}$ on the other hand proposed interband exchange mechanisms for both $J_{1}$ and $J_{2}$ where the exchange of the localized $4 f$ moments is mediated by virtual excitations of chalcogenide-valence band $p$ electrons into the empty $\mathrm{Eu}^{2+} 5 d$ conduction bands, together with a subsequent interband exchange of the $d$ electron ( $p$ hole) with the localized $4 f$ electrons. In this semiconductor analogue of the Ruderman-Kittel-Kasuya-Yosida (RKKY) interaction $J(r) \sim r^{-4}$ is considered for the distance dependencies of the NN and NNN exchange interactions. Both estimates for the distance dependence of the exchange interactions more or less support the picture $1,2,3,89$ of the exchange interactions being continuous functions across different members of the $\mathrm{E} u X$ family. Our analysis clearly shows that the exchange interactions are varying much stronger with the interatomic distances than previously assumed and that the exchange of the anion element can not be attributed solely to shifts in the lattice parameter. With a Grüneisen exponent of $n_{1} \approx 20$, there is a particularly strong dependence of the NN exchange $J_{1}$ as function of the interatomic distances and the exponent $n_{2} \approx 10$ for the distance dependence of the NNN exchange interaction $J_{2}$ coincides with Bloch's 57 empirical law for the volume dependence of the superexchange in magnetic solids.

The possibility to integrate $\mathrm{EuO}$ with $\mathrm{Si}$ and $\mathrm{GaN}^{31,32}$ together with the fact that $T_{C}$ can be increased by doping 33,34 and hydrostatic strain $44,45,90$ to temperatures up to $200 \mathrm{~K}$ led to a renewed interest in the ferromagnetic europium monochalcogenides as possible materials for future spintronic devices. As hydrostatic pressure is not an option for practical applications, epitaxial strain has been suggested as an alternative way to increase the ferromag- 
netic ordering temperature in $\mathrm{EuO}^{91}$. Ingle and Elfimov showed in their ab initio study that biaxial compressive strain increases $T_{C}$ in EuO similarly to the situation in $\mathrm{EuSe}^{11}$ and $\mathrm{EuTe}^{52,53}$, where the antiferromagnetic ordering temperature is increased with a reduction in the in-plane lattice constant of ultrathin (111) oriented epilayers. As shown by Ref. 53, by proper adjustment of the exchange constants, the behavior of epitaxially strained EuTe layers can well be described by our Monte Carlo calculations.

\section{CONCLUSION}

In summary, we have applied the Monte Carlo method to determine the exchange integrals in the $\mathrm{Eu} X$ compounds. To this end, we have determined the general dependences of the magnetic ordering temperatures, i.e., ferromagnetic $T_{C}\left(J_{1}, J_{2}\right)$ and antiferromagnetic $T_{N}\left(J_{1}, J_{2}\right)$ as functions of the NN and NNN exchange interactions of a system of classical Heisenberg spins at the sites of an $f c c$ lattice. This was subsequently applied to determine the distance dependence of the NN and NNN exchange interactions from hydrostatic pres- sure experiments based on the magnetic Grüneisen law ${ }^{57}$ $J_{i}\left(r_{i}\right) \sim r_{i}^{-n_{i}}$, where $r_{i}$ denotes the interatomic distances between neighboring Eu ions. It turns out that the distance dependences of the exchange interactions $J_{1}\left(r_{1}\right)$ and $J_{2}\left(r_{2}\right)$ of the different members of the $\operatorname{Eu} X$ family can be consistently described by Grüneisen exponents $n_{1} \approx 20$ and $n_{2} \approx 10$, where the latter conforms with Bloch's empirical 10/3 law for the volume dependence of the superexchange interaction 57 . The strong dependence of the exchange constants on the lattice parameter provides room for substantially increasing the magnetic ordering temperatures in strained heteroepitaxial structures, which is an important prerequisite for device applications.

\section{Acknowledgments}

This work was supported by the Austrian Science funds FWF and GMe and the Austrian NANO Initiative (NSI). The authors would like to thank Reinhard Folk for usefull hints and valuable disscussions and Daniel Gruber and Johann Messner for technical assistance.
* To whom correspondence should be addressed. E-mail: gunther.springholz@jku.at

1 W. Zinn, J. Magn. Magn. Mater. 3, 23 (1976).

${ }^{2}$ P. Wachter, in Handbook on the Physics and Chemistry of the Rare Earths, edited by K. A. Gschneidner, Jr. and L. Eyring (Elsevier North-Holland, Amsterdam, 1979), vol. 2 , p. 507 .

3 A. Mauger and C. Godart, Phys. Rep. 141, 51 (1986).

${ }^{4}$ T. Kasuya, IBM J. Res. Develop. 14, 214 (1970).

${ }^{5}$ M. S. Seehra and T. M. Giebultowicz, Phys. Rev. B 38, 11898 (1988).

${ }^{6}$ B. T. Matthias, R. M. Bozorth, and J. H. Van Vleck, Phys. Rev. Lett. 7, 160 (1961).

7 T. R. McGuire, B. E. Argyle, M. W. Shafer, and J. S. Smart, Appl. Phys. Lett. 1, 17 (1962).

8 N. F. Oliveira, Jr., S. Foner, Y. Shapira, and T. B. Reed, Phys. Rev. B 5, 2634 (1972).

9 R. Griessen, M. Landolt, and H. R. Ott, Solid State Commun. 9, 2219 (1971).

${ }^{10}$ H. Fukuma, T. Komatsubara, T. Suzuki, E. Kaldis, and T. Kasuya, J. Phys. Soc. Jpn. 54, 3067 (1985).

11 R. T. Lechner, G. Springholz, T. U. Schülli, J. Stangl, T. Schwarzl, and G. Bauer, Phys. Rev. Lett. 94, 157201 (2005).

12 R. Kirchschlager, W. Heiss, R. T. Lechner, G. Bauer, and G. Springholz, Appl. Phys. Lett. 85, 67 (2004).

13 W. Heiss, G. Prechtl, and G. Springholz, Phys. Rev. B 63, 165323 (2001).

14 P. G. Steeneken, L. H. Tjeng, I. Elfimov, G. A. Sawatzky, G. Ghiringhelli, N. B. Brookes, and D.-J. Huang, Phys. Rev. Lett. 88, 47201 (2002).

15 T. S. Santos and J. S. Moodera, Phys. Rev. B 69, 241203 (2004).
16 T. S. Santos, J. S. Moodera, K. V. Raman, E. Negusse, J. Holroyd, J. Dvorak, M. Liberati, Y. U. Idzerda, and E. Arenholz, Phys. Rev. Lett. 101, 147201 (2008).

17 J. S. Moodera, X. Hao, G. A. Gibson, and R. Meservey, Phys. Rev. Lett. 61, 637 (1988).

18 X. Hao, J. S. Moodera, and R. Meservey, Phys. Rev. B 42, 8235 (1990).

19 A. T. Filip, P. LeClair, C. J. P. Smits, J. T. Kohlhepp, H. J. M. Swagten, B. Koopmans, and W. J. M. de Jonge, Appl. Phys. Lett. 81, 1815 (2002).

20 P. LeClair, J. K. Ha, H. J. M. Swagten, J. T. Kohlhepp, C. H. van de Vin, and W. J. M. de Jonge, Appl. Phys. Lett. 80, 625 (2002).

21 C. J. P. Smits, A. T. Filip, J. T. Kohlhepp, H. J. M. Swagten, B. Koopmans, and W. J. M. de Jonge, J. Appl. Phys. 95, 7405 (2004).

22 J. Trbovic, C. Ren, P. Xiong, and S. von Molnár, Appl. Phys. Lett. 87, 082101 (2005).

23 T. Nagahama, T. S. Santos, and J. S. Moodera, Phys. Rev. Lett. 99, 016602 (2007).

24 C. Ren, J. Trbovic, R. L. Kallaher, J. G. Braden, J. S. Parker, S. von Molnár, and P. Xiong, Phys. Rev. B 75, 205208 (2007).

25 J. S. Moodera, R. Meservey, and X. Hao, Phys. Rev. Lett. 70, 853 (1993).

${ }^{26}$ G. Busch, J. Schoenes, and P. Wachter, Solid State Commun. 8, 1841 (1970).

27 J. Schoenes and P. Wachter, Physica B+C 86-88, 125 (1977).

28 M. Suekane, G. Kido, N. Miura, and S. Chikazumi, J. Magn. Magn. Mater. 31-34, 589 (1983).

${ }^{29}$ H. Hori, R. Akimoto, M. Kobayashi, S. Miyamoto, M. Furusawa, N. Kreines, A. Yamagishi, and M. Date, Physica 
B 201, 438 (1994).

30 M. R. Koblischka and R. J. Wijngaarden, Supercond. Sci. Technol. 8, 199 (1995).

31 J. Lettieri, V. Vaithyanathan, S. K. Eah, J. Stephens, V. Sih, D. D. Awschalom, J. Levy, and D. G. Schlom, Appl. Phys. Lett. 83, 975 (2003).

32 A. Schmehl, V. Vaithyanathan, A. Herrnberger, S. Thiel, C. Richter, M. Liberati, T. Heeg, M. Rockerath, L. F. Kourkoutis, S. Muhlbauer, et al., Nature Mater. 6, 882 (2007).

33 T. J. Konno, N. Ogawa, K. Wakoh, K. Sumiyama, and K. Suzuki, Jpn. J. Appl. Phys. 35, 6052 (1996).

${ }^{34}$ H. Ott, S. J. Heise, R. Sutarto, Z. Hu, C. F. Chang, H. H. Hsieh, H.-J. Lin, C. T. Chen, and L. H. Tjeng, Phys. Rev. B 73, 94407 (2006).

35 D. B. McWhan, P. C. Souers, and G. Jura, Phys. Rev. 143, 385 (1966).

36 V. C. Srivastava and R. Stevenson, Can. J. Phys. 46, 2703 (1968).

37 U. F. Klein, G. Wortmann, and G. M. Kalvius, J. Magn. Magn. Mater. 3, 50 (1976).

38 U. F. Klein, J. Moser, G. Wortmann, and G. M. Kalvius, Physica B 86-88, 118 (1977).

39 J. Moser, G. Wortmann, N. Bykovetz, and G. M. Kalvius, J. Magn. Magn. Mater. 12, 77 (1979).

${ }^{40}$ H. Fujiwara, H. Kadomatsu, M. Kurisu, T. Hihara, K. Kojima, and T. Kamigaichi, Solid State Commun. 42, 509 (1982).

41 C. Sauer, A. Zaker, and W. Zinn, J. Magn. Magn. Mater. 31-34, 423 (1983).

42 T. Hihara, K. Hiraoka, K. Kojima, T. Kamigaichi, and T. Kino, J. Magn. Magn. Mater. 52, 443 (1985).

43 V. G. Tissen and E. G. Ponyatovskii, JETP Lett. 46, 361 (1987).

44 J. Moser, G. M. Kalvius, and W. Zinn, Hyperfine Interact. 41, 499 (1988).

45 M. M. Abd-Elmeguid and R. D. Taylor, Phys. Rev. B 42, 1048 (1990).

46 I. N. Goncharenko and I. Mirebeau, Europhys. Lett. 37, 633 (1997).

47 M. Ishizuka, Y. Kai, R. Akimoto, M. Kobayashi, K. Amaya, and S. Endo, J. Magn. Magn. Mater. 166, 211 (1997).

48 I. N. Goncharenko and I. Mirebeau, Phys. Rev. Lett. 80, 1082 (1998).

49 G. Springholz and G. Bauer, Phys. Rev. B 48, 10998 (1993).

50 N. Frank, G. Springholz, and G. Bauer, Phys. Rev. Lett. 73, 2236 (1994)

51 A. Stachow-Wójcik, T. Story, W. Dobrowolski, M. Arciszewska, R. R. Gałazka, M. W. Kreijveld, C. H. W. Swüste, H. J. M. Swagten, W. J. M. de Jonge, A. Twardowski, et al., Phys. Rev. B 60, 15220 (1999).

${ }^{52}$ H. Kępa, G. Springholz, T. M. Giebultowicz, K. I. Goldman, C. F. Majkrzak, P. Kacman, J. Blinowski, S. Holl, H. Krenn, and G. Bauer, Phys. Rev. B 68, 24419 (2003).

53 E. Schierle, E. Weschke, A. Gottberg, W. Söllinger, W. Heiss, G. Springholz, and G. Kaindl, Phys. Rev. Lett. 101, 267202 (2008).

${ }^{54}$ I. N. Goncharenko, I. Mirebeau, and A. Ochiai, Hyperfine Interactions 128, 225 (2000).

55 D. P. Landau and K. Binder, A Guide to Monte Carlo Simulations in Statistical Physics (Cambridge University Press, 2000).
56 S. M. Scott and T. M. Giebultowicz, J. Appl. Phys. 91, 8724 (2002).

57 D. Bloch, J. Phys. Chem. Solids 27, 881 (1966).

58 G. Springholz and G. Bauer, Appl. Phys. Lett. 62, 2399 (1993).

59 N. Metropolis, A. W. Rosenbluth, M. N. Rosenbluth, A. H. Teller, and E. Teller, J. Chem. Phys. 21, 1087 (1953).

60 D. S. Ritchie and M. E. Fisher, Phys. Rev. B 5, 2668 (1972).

61 P. Peczak, A. M. Ferrenberg, and D. P. Landau, Phys. Rev. B 43, 6087 (1991).

${ }^{62}$ K. Chen, A. M. Ferrenberg, and D. P. Landau, Phys. Rev. B 48, 3249 (1993).

63 R. M. Nowotny and K. Binder, Z. Phys. B 77, 287 (1989).

${ }^{64}$ W. Nolting, Quantentheorie des Magnetismus, vol. 2 (B. G. Teubner, Stuttgart, 1986).

65 P. W. Anderson, Phys. Rev. 83, 1260 (1951).

${ }^{66}$ W. Heiss, G. Prechtl, and G. Springholz, Appl. Phys. Lett. 78, 3484 (2001).

67 W. Heiss, R. Kirchschlager, G. Springholz, Z. Chen, M. Debnath, and Y. Oka, Phys. Rev. B 70, 35209 (2004).

68 M. E. Fisher, Philos. Mag. 7, 1731 (1962).

69 U. Köbler, I. Apfelstedt, K. Fischer, W. Zinn, E. Scheer, J. Wosnitza, H. v. Löhneysen, and T. Brückel, Z. Phys. B 92, 475 (1993)

70 J. Kuneš, W. Ku, and P. E. Warren, J. Phys. Soc. Jap. 74, 1408 (2005).

71 V.-C. Lee and L. Liu, Phys. Rev. B 30, 2026 (1984).

72 D. I. Khomskii and G. A. Sawatzky, Solid State Commun. 102, 87 (1997).

73 A. Radomska and T. Balcerzak, Phys. Status Solidi B 225, 229 (2001).

74 L. Passell, O. W. Dietrich, and J. Als-Nielsen, Phys. Rev. B 14, 4897 (1976).

75 H. G. Bohn, W. Zinn, B. Dorner, and A. Kollmar, J. Appl. Phys. 52, 2228 (1981).

76 H. A. Mook, Phys. Rev. Lett. 46, 508 (1981).

77 B. C. Passenheim, D. C. McCollum, Jr., and J. Callaway, Phys. Lett. 23, 634 (1966).

78 J. A. J. Henderson, G. R. Brown, T. B. Reed, and H. Meyer, Journal of Applied Physics 41, 946 (1970).

79 P. K. Schwob and G. E. Everett, J. Phys. Colloques 32 C1 (1971).

${ }^{80}$ O. W. Dietrich, A. J. Henderson, and H. Meyer, Phys. Rev. B 12, 2844 (1975).

81 S. H. Charap and E. L. Boyd, Phys. Rev. 133, A811 (1964).

82 E. L. Boyd, Phys. Rev. 145, 174 (1966).

83 A. Comment, J.-P. Ansermet, C. P. Slichter, H. Rho, C. S. Snow, and S. L. Cooper, Phys. Rev. B 72, 014428 (2005).

84 P. K. Schwob, M. Tachiki, and G. E. Everett, Phys. Rev. B 10, 165 (1974).

85 S. Heathman, T. Le Bihan, S. Darracq, C. Abraham, D. J. A. De Ridder, U. Benedict, K. Mattenberger, and O. Vogt, J. Alloys and Compounds 230, 89 (1995).

86 S. Blundell, Magnetism in Condensed Matter, Oxford Master Series in Condensed Matter Physics (Oxford University Press, 2001).

87 W. Heitler and F. London, Z. Phys. A 44, 455 (1927).

88 R. E. Coffman and G. R. Buettner, J. Phys. Chem. 83, 2387 (1979).

89 T. R. McGuire and M. W. Shafer, J. Appl. Phys. 35, 984 (1964).

90 D. DiMarzio, M. Croft, N. Sakai, and M. W. Shafer, Phys. Rev. B 35, 8891 (1987). 
91 N. J. C. Ingle and I. S. Elfimov, Phys. Rev. B 77, 121202 (2008). 